\title{
Metric Currents and Geometry of Wasserstein Spaces
}

\author{
LUCA GRANIERI (*)
}

ABSTRACT - We investigate some geometric aspects of Wasserstein spaces through the continuity equation as worked out in mass transportation theory. By defining a suitable homology on the flat torus $T^{n}$, we prove that the space $\mathcal{P}_{p}\left({ }^{\top} T^{n}\right)$ has nontrivial homology in a metric sense. As a byproduct of the developed tools, we show that every parametrization of a Mather's minimal measure on " $T^{n}$ corresponds to a mass minimizing metric current on $\mathcal{P}_{p}\left({ }^{(} T^{n}\right)$ in its homology class.

\section{Introduction.}

\section{1 - The Monge-Kantorovich problem.}

Optimal transport problems, also known as Monge-Kantorovich problems, have been very intensively studied in the last 10 years and, due to the numerous and important applications to PDE, shape optimization and Calculus of Variations, we witnessed a spectacular development of the field. For the details of this theory, the interested reader may look at the book and lecture notes [1, 7, 31,32], the paper [17] and for some of the applications [6, 22]. Our description will be restricted to the setting of a compact Riemannian manifold $M$ without boundary, however many of the concepts of this section could be formulated in general metric spaces.

Let $c: M \times M \rightarrow \mathbb{R}^{+}$be a Borel function. The Monge problem is formulated as follows: given two probability measures $\mu, v$ find a map $t: M \rightarrow M$ such that $t_{\sharp} \mu=v$ ( $\sharp$ denotes the push-forward of measures) and such that $t$ minimizes

$$
\int_{M} c(x, t(x)) d \mu
$$

(*) Indirizzo dell'A.: Dipartimento di Matematica Politecnico di Bari, via Orabona 4, 70125 Bari, Italy

E-mail: l.granieri@poliba.it, granieriluca@libero.it MSC 2000. 37J50, 49Q20, 49Q15. 
among the maps with the same property. It may happens that the set of admissible maps is empty (e.g. $\mu=\delta_{x}$ and $v=\frac{1}{2}\left(\delta_{y}+\delta_{z}\right)$ ). Then the problem is reformulated in its Kantorovich's relaxation. Find $\gamma \in \mathcal{P}(M \times M)$ such that $\pi_{\sharp}^{1} \gamma=\mu$ and $\pi_{\sharp}^{2} \gamma=v\left(\pi^{1}\right.$ and $\pi^{2}$ are the projection on factors of $M \times M)$ and such that $\gamma$ minimizes

$$
\int_{M \times M} c(x, y) d \gamma(x, y) .
$$

If $t$ is admissible for the Monge problem then the measure associated in the usual way to the graph of $t$, i.e. $\gamma=(I \times t)_{\sharp} \mu$, is admissible for the Kantorovich problem. However the class of admissible measures for the Kantorovich problem is never empty as it contains $\mu \otimes v$. Moreover, the Kantorovich problem is linear. Existence of minimizers for the Monge problem is difficult and may fails, while for the Kantorovich problem semicontinuity of $c$ is enough.

If $c$ is a distance then the cost

$$
d_{c}(\mu, v)=\min \left\{\int_{M \times M} c(x, y) d \gamma(x, y): \pi_{\sharp}^{1} \gamma=\mu, \pi_{\sharp}^{2} \gamma=v\right\}
$$

defines a distance on $\mathcal{P}(M)$. If $d$ is the geodesic distance of the manifold then for $p \geq 1$ also

$$
W_{p}(\mu, v)=\left(\min \left\{\int_{M \times M} d^{p}(x, y) d \gamma(x, y): \pi_{\sharp}^{1} \gamma=\mu, \pi_{\sharp}^{2} \gamma=v\right\}\right)^{1 / p}
$$

defines a distance on $\mathcal{P}(M)$ called Wasserstein distance. Moreover, $\left(\mathcal{P}(M), W_{p}\right)$ is complete and $W_{p}$ metrizes the weak* convergence of measures. The metric structure induced on $\mathcal{P}(M)$ is extremely rich and will play an important role in this paper. In particular $\mathcal{P}_{p}(M):=\left(\mathcal{P}(M), W_{p}\right)$ admits a tangent space. We will get in details in a subsection of preliminary results.

Finally, whenever $c(x, y)$ is a "length" cost, which means that there exists a Lagrangian $L: T M \rightarrow \mathbb{R}^{+}$such that

$$
c(x, y)=\inf _{\substack{\gamma \in A C(0,1), M \\ \gamma(0)=x, \gamma(1)=y}} \int L(\gamma(t), \dot{\gamma}(t)) d t,
$$

then the transport problem has a third formulation due to Brenier. The basic idea is to look at paths connecting the two measures $\mu, v \in \mathcal{P}(M)$ that 
we want to transport. If, in the simplest case of $\mu=\delta_{x}$ and $v=\delta_{y}$, we take a regular curve $\gamma$ connecting $x$ to $y$, then the measure $\rho_{t}=\delta_{\gamma(t)}$ and the velocity field $v_{t}=\dot{\gamma}(t)$ are related by the equation

$$
\frac{\partial \rho}{\partial t}+\nabla \cdot\left(v_{t} \rho_{t}\right)=0 \quad \text { on }(0,1) \times M,
$$

in the sense of distribution. Indeed, if we take a test function $\varphi(t, x) \in \mathcal{C}_{c}^{\infty}((0,1) \times M)$, we have

$$
\begin{aligned}
& \left(\frac{\partial \rho}{\partial t}+\nabla \cdot\left(v_{t} \rho_{t}\right)\right)(\varphi)= \\
& =-\int_{0}^{1} \int_{M} \partial_{t} \varphi(t, x) d \rho_{t} d t-\int_{0}^{1} \int_{M}\left\langle d_{x} \varphi(t, x), v_{t}\right\rangle d \rho_{t} d t= \\
& =-\int_{0}^{1}\left[\partial_{t} \varphi(t, \gamma(t))+\left\langle d_{x} \varphi(\gamma(t)), \dot{\gamma}(t)\right\rangle\right] d t=-\int_{0}^{1} \frac{d}{d t}[\varphi(t, \gamma(t))] d t=0 .
\end{aligned}
$$

More generally, we can consider couples $\left(\rho_{t}, v(x, t)\right) \in \mathcal{P}(M) \times \mathcal{M}^{n}(M)$ which satisfy the equation (1.1) as admissible configurations. In this setting, Brenier's formulation of the optimal mass transportation problem amounts to minimize

$$
\int_{0}^{1} \int_{M} L(x, v(x, t)) d \rho_{t}(x) d t
$$

among the pair $(\rho, v)$ where $\rho:[0,1] \rightarrow \mathcal{P}(M)$, and for each $t, v(\cdot, t)$ is a tangent vector field on $M$ defined $\rho_{t}$ almost everywhere, which satisfies the continuity equation:

$$
\left\{\begin{array}{l}
\frac{\partial \rho}{\partial t}+\nabla \cdot(v \rho)=0 \quad \text { on }(0,1) \times M, \\
\rho(0)=\mu, \quad \rho(1)=v .
\end{array}\right.
$$

We remark that if $\int_{0}^{1} \int_{M}\left\|v_{t}\right\| d \rho_{t} d t<+\infty$, then for every $\varphi \in \mathcal{C}^{\infty}(M)$ the map $t \mapsto \int_{0}^{1} \varphi d \rho_{t} \in W_{l o c}^{1,1}(0,1)$. Actually, it turns out that $\rho_{t}$ is an absolutely continuous (a.c.) curve in the space $\mathcal{P}(M)$ endowed with the 1-Wasserstein distance. 


\section{2 - Mather's minimal measures and Eulerian representations.}

A measure $\mu \in \mathcal{M}(T M)$ is said to be closed if for all exact forms $\omega$ it results

$$
\int_{T M}\langle\omega(x), v\rangle d \mu=0
$$

According to this definition we set

$$
\mathcal{M}^{c}=\left\{\mu \in \mathcal{M}(T M) \mid \mu \text { is closed, } \mu \in \mathcal{P}(T M), \int_{T M} L(x, v) d \mu<+\infty\right\} .
$$

To each measure $\mu \in \mathcal{M}^{c}$ we can associate the homology class of $\mu$ which we will denote by $[\mu] \in H_{1}(M, \mathbb{R})$ (by duality with $H^{1}$ ). Indeed, thanks to the fact that $L$ is superlinear and $\int_{T M} L(x, v) d \mu<+\infty, \mu$ acts in a natural way on the set of the closed 1-forms on $M$ by

$$
\omega \mapsto \int_{T M}\langle\omega(x), v\rangle d \mu
$$

and thanks to condition (1.3), this action passes to the quotient by the exact forms.

Once we fix an homology class $[h]$, Mather's variational problem amounts to:

$$
\text { Minimize }\left\{\int_{T M} L(x, v) d \mu \mid \mu \in \mathcal{M}^{c},[\mu]=[h]\right\} .
$$

A remarkable property of problem $\mathbf{M}$ is the following: we minimize an action functional which depends on $L$ on measures which are merely closed. However, it turns out that the minimal measures are also invariant for the flow associated to the Lagrangian $L$, see for example $[5,10]$.

There is a deep connection between Mather's problem and the MongeKantorovich theory (see [9, 11, 19, 20]). In particular, in [11] is considered the following problem: minimize the convex and l.s.c. functional

$$
\mathcal{K}(\rho, v)=\int_{0}^{1} \int_{M} L(x, v(x, t)) d \rho_{t} d t
$$


among all pairs $\rho:[0,1] \rightarrow \mathcal{P}(M)$ and $v: M \times[0,1] \rightarrow T M$ which solve

$$
\left\{\begin{array}{l}
\frac{\partial \rho}{\partial t}+\nabla \cdot(v \rho)=0 \quad \text { in }(0,1) \times M \\
\rho(0)=\rho(1)
\end{array}\right.
$$

and satisfy an homological constraint in a sense that will be explained right below. To each solution $(\rho, v)$ of (1.5) we can associate a closed, normal 1current

$$
T_{\rho, v}: \omega \mapsto \int_{0}^{1} \int_{M}\langle\omega(x), v(x, t)\rangle d \rho_{t} d t .
$$

As already noticed $T_{\rho, v}$ has a well defined homology class. Then the problem is formulated as follows

(P) Minimize $\left\{\mathcal{K}(\rho, v) \mid(\rho, v)\right.$ satisfies (1.5) and $\left.\left[T_{\rho, v}\right]=[h]\right\}$.

It turns out that problem $\mathbf{P}$ is equivalent to the Mather's problem $\mathbf{M}$. Moreover, to every Mather's minimal measure one can associate an optimal couple $(\rho, v)$ of $\mathbf{P}$ and vice-versa. Each minimizing pair $(\rho, v)$ will be called an Eulerian representation of the current $T_{\rho, v}$ and of the corresponding Mather's minimal measure.

Notice that different Eulerian representations can be associated to the same current or measure.

Finally, in the case $L(x, v)=|v|^{p}$ problem $\mathbf{P}$ is equivalent to the classical problem of minimizing the mass (to the power $p$ ) of closed 1-currents in a given homology class (see [5, 11]).

\section{3 - Description of the results.}

Every Eulerian representation $(\rho, v)$ of a Mather's minimal measure leads naturally to a closed 1-metric currents on a Wasserstein space (see the next section for definitions and main properties). It is natural to ask whether these Eulerian representations enjoy metric minimality properties or not. To make sense to this question we need some tools able to recover that two closed metric 1-currents are homologous on $\mathcal{P}_{p}(M)$.

The matter is to specify the meaning of homology classes in a metric framework. Since a deep theory of metric currents is already available in 
[3], a natural way is to define metric currents $T$ and $S$ to be homologous if there exists a metric 2-current $N$ such that $T-S=\partial N$. Regarding this metric definition of homology, at the best of our knowledge, it seems that all the tools available in the literature rely on some cone construction as in $[3,33,34]$, or by a differential form approach as in [16]. In any case, these methods are relative to metric spaces with trivial homology in the metric sense, and they are not applicable to general Wasserstein spaces $\mathcal{P}_{p}(M)$. In Section 3 we show that also on nice spaces such as Hadamard spaces $M$, we cannot say in general too much about the homology of $\mathcal{P}_{p}(M)$. Indeed, we are just able to show that all the closed Lipschitz curves $\delta_{\gamma(t)}$ on $\mathcal{P}_{p}(M)$, corresponding to closed cycles $\gamma$ of the Hadamard space $M$, are homologous to zero. This fact is proved by considering barycenter maps. However, the existence of a barycenter map imposes many restrictions to the geometry of $M$ (see Theorem 3.3). As a byproduct, this discussion furnishes a counterexample to the extension of Lipschitz maps between metric spaces (see Example 3.5).

Although $\mathcal{P}_{p}(M)$ is always contractible, see Proposition 3.1, it is known that $\mathcal{P}_{p}(M)$ has trivial homology only for special geometry of the underlying space $M$ (see [16, 34]). In Section 4 and Section 5 we propose to investigate the metric homology of $\mathcal{P}_{p}(M)$ by means of mass transportation tools. More precisely, by using the continuity equation (1.2) we associate an euclidean current on $M$ to every metric current on $\mathcal{P}_{p}(M)$ by $\mathrm{a} \sim$ operator defined by equation (4.3). It turns out that this operator well relates metric 1-currents to metric 0-currents as stated in Theorem 4.5. Unfortunately, we are not able to prove the same well behavior for the analogous $\sim$ operator which relates metric 2-currents to metric 1-currents. However, on the flat torus ${ }^{T}{ }^{n}$ some of the program can be carried on. In particular, we restrict the homology by considering metric 2currents which satisfy the compatibility condition (5.8). It results that to every distinct homology classes on $T^{n}$ correspond distinct such restricted metric homology classes on $\mathcal{P}_{p}\left(T^{n}\right)$ (see Lemma 5.4). Therefore, the space $\mathcal{P}_{p}\left(T^{n}\right)$ is a first example of a metric space with non-trivial homology in a metric sense. Once the question of finding homology classes in a metric sense has some answer, although partial, it is meaningful to address the question of recovering mass minimizing currents on $\mathcal{P}_{p}(M)$ by knowing the structure of the Eulerian representations of Mather's measures on the underlying space $M$. As a byproduct of the developed tools, in Section 5.3 we show that to every Mather's minimal Eulerian representation $(\rho, v)$ corresponds a mass minimizing metric 1-current on $\mathcal{P}_{p}\left(T^{n}\right)$ in its homology class. 


\section{Preliminary results.}

\section{1 - Normal 1-currents.}

This section collects some definitions and technical facts about normal 1-currents and their representations which will be used explicitly in the paper or which are useful to give sense to some definitions. The exposition is adapted to the fact that the manifold $M$ is compact and then different from what would be on an open subset $\Omega$ of $\mathbb{R}^{N}$.

By $\Gamma^{\infty}\left(T^{*} M\right)$ we denote the space of $\mathcal{C}^{\infty}$, 1-dimensional forms. $\Gamma^{\infty}\left(T^{*} M\right)$ is usually equipped with the norm $\|\omega\|_{\infty}=\sup _{M}|\omega(x)|$. The space of normal, 1-dimensional currents is the space of linear, continuous (as well the boundary) functionals on $\Gamma^{\infty}\left(T^{*} M\right)$ and is denoted by $\mathcal{N}_{1}(M)$.

The subspace of the currents $T \in \mathcal{N}_{1}(M)$ such that $T(\omega)=0$ whenever $\omega$ is an exact form is the space of normal, closed 1-currents and it is denoted by $\mathcal{N}_{1}^{c}(M)$.

The mass norm of a normal current (in short: "mass") is defined as follows:

$$
M(T)=\sup \left\{T(\omega) \mid\|\omega\|_{\infty} \leq 1\right\}
$$

The boundary of a current is defined by duality with the differential through the formula:

$$
T(d \phi)=\partial T(\phi) \text { for all } \phi \in \mathcal{C}^{\infty}(M)
$$

Then for a current $T$, being closed is equivalent to $\partial T=0$.

Any $T \in \mathcal{N}_{1}(M)$ can be represented by integration using a probability measure $\sigma$ on $M$ and a tangent vector field $X$ defined $\sigma$-a.e. as follows:

$$
T(\omega)=\int_{M}\langle\omega(x), X\rangle d \sigma .
$$

There are many references in the literature for the representation of normal currents, among them we refer to [15] or Theorem 1 in section 2.3 of [18].

\section{2 - Currents in metric spaces.}

In this section we introduce the notion of (normal, rectifiable, integer) currents in a metric space $E$, according to the paper [3], who first contains 
a complete treatment of the subject. We will apply this theory in Section 5 for the Wasserstein space $\mathcal{P}_{p}(M)$. Since we are mostly interested in 0,1 and 2-currents we confine ourselves to state the principal results regarding these particular (metric) functionals.

DEFinition 2.1. A k-current $T, k=0,1,2$, is a multilinear functional on the set of $(k+1)$-couples $\left(f_{0}, f_{1}, \ldots, f_{k}\right) \in \operatorname{Lip}(E)^{k+1}$, with $f_{0} \in L^{\infty}(E)$, satisfying the following properties:

i) $T$ is continuous along sequences $\left(f_{0}, f_{1}^{n}, \ldots, f_{k}^{n}\right)$ converging pointwise and with $\sup _{n} \operatorname{Lip}\left(f_{i}^{n}\right)<+\infty$;

ii) $T$ is local, in the sense that $T\left(f_{0}, f_{1}, \ldots, f_{k}\right)=0$ if $f_{i}$ is constant for some $i \in\{1, \ldots, k\}$ in a neighborhood of $f_{0} \neq 0$;

iii) $T$ has finite mass, i.e. there exist $\mu \in \mathcal{M}(E)$ such that $\left|T\left(f_{0}, f_{1}, \ldots, f_{k}\right)\right| \leq \Pi_{i=1}^{k} \operatorname{Lip}\left(f_{i}\right) \int_{E}\left|f_{0}\right| d \mu$

The least measure $\mu$ satisfying iii) is said mass of $T$ and it is denoted by $\|T\|$, while $M(T):=\|T\|(E)$ is the total mass of $T$.

REMARK 2.2. Given a $k$-current $T$, its total mass can be computed also as

$M(T)=\sup \left\{\sum_{i=1}^{N}\left|T\left(\chi_{B_{i}}, f_{1}^{i}, \ldots, f_{k}^{i}\right)\right|:\left\{B_{i}\right\}_{i}\right.$ Borel partition of $\left.E, f_{k}^{i} \in \operatorname{Lip}_{1}(E)\right\}$,

where $\operatorname{Lip}_{1}(E)$ is the set of Lipschitz functions with Lipschitz constant $\operatorname{Lip}(f)$ not greater than 1 . Observe that the metric functional $T$ could be extended on $k$-tuples with the first argument a merely bounded function. For $k$-currents it is possible to define a boundary operator $\partial T$ as follows

$$
\partial T\left(f_{0}, f_{1}, \ldots, f_{k-1}\right):=T\left(1, f_{0}, f_{1}, \ldots, f_{k-1}\right) .
$$

The restriction $T\llcorner A$ of $T$ to a Borel set $A \subset E$ is given by

$$
T\left\llcorner A\left(f_{0}, f_{1}, \ldots, f_{k}\right):=T\left(f_{0} \chi_{A}, f_{1}, \ldots, f_{k}\right) .\right.
$$

If $\varphi \in \operatorname{Lip}(E, F)$, with $F$ another metric space, we define also the pushforward of $T$, as $\varphi_{\#}(T)\left(f_{0}, f_{1}, \ldots, f_{k}\right):=T\left(f_{0} \circ \varphi, f_{1} \circ \varphi, \ldots, f_{k} \circ \varphi\right)$. Notice that the boundary and the push-forward operator commute. The pushforward operator will be very useful since every integer rectifiable current will be generated by Lipschitz images of euclidean metric currents as below, with $g \in L^{1}\left(\mathbb{R}^{k}, Z\right)$. 
Examples of 1-currents for $E=\mathbb{R}$, and 2-currents if $E=\mathbb{R}^{2}$, can be given once fixed a function $g \in L^{1}(\mathbb{R})$ or $g \in L^{1}\left(\mathbb{R}^{2}\right)$ in the following way:

$$
\begin{aligned}
& T_{g}\left(f_{0}, f_{1}\right):=\int_{\mathbb{R}} g(x) f_{0}(x) f_{1}^{\prime}(x) d x \\
& N_{g}\left(f_{0}, f_{1}, f_{2}\right):=\int_{\mathbb{R}^{2}} g(x) f_{0}(x) \operatorname{det}\left(\nabla f_{1}, \nabla f_{2}\right) d x .
\end{aligned}
$$

We'll come back to these fundamental examples to define integer currents and also in view of the representation Theorem 2.4.

Even if it will be used only to introduce rectifiable currents, we define countably $\mathcal{H}^{k}$-rectifiable sets as sets $S$ such that

$$
\mathcal{H}^{k}\left(S \backslash \bigcup_{i=0}^{\infty} h_{i}\left(K_{i}\right)\right)=0
$$

for $K_{i}$ compact sets and $h_{i}: K_{i} \rightarrow E$ bi-Lipschitz functions.

We can now introduce the notion of normal, rectifiable and integer $k$ currents.

DEFINITION 2.3. A $k$-current $T$ will be said normal if $\partial T$ is still a $(k-1)$ current.

A $k$-current $T$ will be said integer rectifiable if $\|T\|$ is concentrated on a countably $\mathcal{H}^{k}$-rectifiable set and, for any $\varphi \in \operatorname{Lip}\left(E, \mathbb{R}^{k}\right)$ and for any open set $A$ of $E, \varphi_{\#}\left(T\llcorner A)=T_{g}\right.$ (see representation in (2.1)) with $g \in L^{1}\left(\mathbb{R}^{k}, Z\right)$. The set of integer rectifiable $k$-currents will be denoted as $\mathcal{I}_{k}(E)$ while $\mathcal{N}_{k}(E)$ will be the set of normal $k$-currents. Finally, we denote by $\boldsymbol{I}_{k}(E)$ the space of integer rectifiable normal currents.

If $k=1,2$ any $k$-current in $\mathbb{R}^{k}$ can be represented, uniquely, as in (2.1); moreover, if $T$ is normal the function $g$ turns out to be $B V\left(\mathbb{R}^{k}\right)$.

Finally we state the result that allows to decompose any integer rectifiable currents as sums of Lipschitz push-forward of integer euclidean metric currents.

THEOREM 2.4. $T \in \mathcal{I}_{k}(E)$ if and only if there exist compact sets $K_{i} \subset \mathbb{R}^{k}, \theta_{i} \in L^{1}\left(\mathbb{R}^{k}, Z\right)$ and bi-Lipschitz functions $h_{i}: K_{i} \rightarrow E$ with $\operatorname{supp}\left(\theta_{i}\right) \subset K_{i}$ such that $T=\sum_{i=1}^{\infty} h_{i \#}\left(T_{\theta_{i}}\right)$ and $M(T)=\sum_{i=1}^{\infty} M\left(h_{i \#}\left(T_{\theta_{i}}\right)\right)$.

For normal currents it turns out that the functions $\theta_{i}$ of Theorem 2.4 are actually $B V$ functions, see Theorem 3.7 of [3]. In the case $k=1$, and 
considering a geodesic space $E$ (see Section 2.5), the Lipschitz function $h_{i}: K_{i} \rightarrow E$ can be extended in a Lipschitz way to intervals such that $K_{i} \subset\left[a_{i}, b_{i}\right]$. As a reference for extension of Lipschitz maps we refer for instance to [12]. Therefore, in such a case Theorem 2.4 yields the representation $T=\sum_{i=1}^{\infty} T_{\theta_{i}, \rho_{i}}$ where $\rho_{i}:\left[a_{i}, b_{i}\right] \rightarrow E$ is a Lipschitz function while the current $T_{\theta_{i}, \rho_{i}}$ is defined by

$$
T_{\theta_{i}, \rho_{i}}\left(f_{0}, f_{1}\right)=\int_{a_{i}}^{b_{i}} \theta_{i}(t) f_{0}\left(\rho_{i}(t)\right) \frac{d}{d t} f_{1}\left(\rho_{i}(t)\right) d t .
$$

The definition of $\mathcal{I}_{k}(E)$ allows us to introduce the homology of $E$ as follows. Consider the chains induced by the boundary operator $\partial$ (observe that $\partial^{2}=0$ )

$$
\cdots \mathcal{I}_{k+1}(E) \stackrel{\partial}{\rightarrow} \mathcal{I}_{k}(E) \stackrel{\partial}{\rightarrow} \mathcal{I}_{k-1}(E) \stackrel{\partial}{\rightarrow} \cdots
$$

we define the k-homology as

$$
H_{k}(E)=\frac{\operatorname{Ker} \partial_{\mathcal{I}_{k}}}{\operatorname{Im} \partial_{\mathcal{I}_{k+1}}}
$$

In particular we will use the homology of $\mathcal{P}_{p}(M)$.

\section{3 - Kantorovich duality.}

In this section we recall some useful duality results for the MongeKantorovich problem. The following Theorem can be found in [31].

TheORem 2.5. Let $c(x, y)$ a positive continuous cost on $M$. Then the minimum value of the Kantorovich problem is equal to

$$
\sup _{\varphi, \psi \in \mathcal{C}(M, \mathbb{R})}\left\{\int_{M} \varphi(x) d f^{+}+\int_{M} \psi(y) d f^{-} \mid \varphi(x)+\psi(y) \leq c(x, y)\right\} .
$$

If $c$ is the geodesic distance $d$ on $M$, duality formulation becomes:

THEOREM 2.6. If $c(x, y)=d(x, y)$ then the minimum value of the Kantorovich problem is equal to

$$
\sup \left\{\int_{M} u d\left(f^{+}-f^{-}\right) \mid u \in \operatorname{Lip}_{1}(M, d)\right\} .
$$


Actually, the supremum in Theorem 2.6 is attained and the optimal $u$ are called Kantorovich potentials.

\section{4 - The continuity equation and the tangent space to $\mathcal{P}_{p}(M)$.}

The continuity equation (1.2) has been used in the Monge-Kantorovich theory since its beginning for many applications. The fact that it characterizes the a.c. curves on the space of probability measures equipped with the Wasserstein metric was only recently pointed out and the full proof is contained in [2]. Here we summarize some results from that paper. For an analysis in metric spaces we refer to [2, 4]. For Lipschitz function on a metric space $(M, d)$ we introduce the metric derivative according to the following definition.

Definition 2.7. Given a curve $\rho:[a, b] \rightarrow(M, d)$ we define the metric derivative at the point $t \in] a, b[$ as the limit

$$
\lim _{h \rightarrow 0} \frac{d(\rho(t+h), \rho(t))}{h}
$$

whenever it exists and in this case we denote it by $|\dot{\rho}|(t)$.

If $\rho:[a, b] \rightarrow(M, d)$ is a Lipschitz curve, by metric Rademacher Theorem the metric derivative of $\rho$ exists at $\mathcal{L}^{1}$-a.e. point in $[a, b]$. Furthermore, the length of the Lipschitz curve $\rho$ is given by

$$
l(\rho)=\int_{a}^{b}|\dot{\rho}|(t) d t .
$$

We restrict our treatment to the case of $\mathcal{P}_{p}(M):=\left(\mathcal{P}(M), W_{p}\right)$ for $p \geq 1$. The tangent space to $\mathcal{P}_{p}(M)$ at a point $\mu$ is defined as follows:

$$
\operatorname{Tan}_{\mu} \mathcal{P}_{p}(M):={\overline{\left\{J_{q}(\nabla \varphi) \varphi \in C^{\infty}(M)\right\}_{L^{p}(\mu)}}}
$$

where $q$ is the dual exponent of $p$ and $J_{q}: L^{q}(\mu) \rightarrow L^{p}(\mu)$ denotes the duality map

$$
J_{q}(v)=\frac{|v|^{q-2} v}{\|v\|_{L^{q}(\mu)}^{q-2}} .
$$

The following theorem relates a. c. curves in $\mathcal{P}_{p}(M)$ to the continuity equation and, in some sense, justifies the definition of the tangent space. 
Theorem 2.8. Let $\rho:[0,1] \rightarrow \mathcal{P}_{p}(M)$ be a curve. If $\rho$ is a.c. and $|\dot{\rho}| \in L^{1}(0,1)$ is its metric derivative, then there exists a Borel vector field $v:(t, x) \mapsto v_{t}(x)$ such that

$$
v_{t} \in L^{p}\left(\rho_{t}\right) \text { and }\left\|v_{t}\right\|_{L^{p}\left(\rho_{t}\right)} \leq|\dot{\rho}|(t) \text { for } \mathcal{L}^{1}-\text { a.e. } t \in[0,1]
$$

and the continuity equation

$$
\frac{\partial \rho}{\partial t}+\nabla \cdot v \rho=0 \text { in } \quad(0,1) \times M
$$

is satisfied in the sense of distributions. Moreover for a.e. $t \in(0,1) v_{t}$ belongs to $T_{\rho(t)} \mathcal{P}_{p}(M)$.

Conversely, if $\rho$ satisfies the continuity equation for some vector fields $v_{t}$ such that $\left\|v_{t}\right\|_{L^{p}\left(\rho_{t}\right)} \in L^{1}(0,1)$, then $t \mapsto \rho(t)$ is a.c. and

$$
|\dot{\rho}|(t) \leq\left\|v_{t}\right\|_{L^{p}\left(\rho_{t}\right)} \text { for } \mathcal{L}^{1}-\text { a.e. } t \in[0,1] .
$$

REMARK 2.9. The minimality property (2.4) uniquely determines a tangent field $v_{t}$. We will refer to $v_{t}$ as the tangent vector associated to the curve $\rho$.

There are also previous definitions of tangent space to $\mu$ in $\mathcal{P}_{p}(M)$, see for example [26]. In [2] there is also an infinitesimal characterization of the tangent space in terms of transport maps.

\section{5 - Basic properties of Wasserstein distances.}

Since we are interested to study the space $\mathcal{P}(M)$ with $M$ a compact Riemannian manifold, throughout this paper $(M, d)$ is assumed to be a complete, separable and geodesic space. A metric space is said geodesic if for each pair of points $x, y \in M$ the distance $d(x, y)$ is given by

$$
d(x, y)=\min \left\{l(\gamma)=\int_{0}^{1}|\dot{\gamma}|(t) d t \mid \gamma \in \operatorname{Lip}([0,1], M), \gamma(0)=x, \gamma(1)=y\right\} .
$$

Where $|\dot{\gamma}|$ is the metric derivative of $\gamma$ defined by (2.3). If $x, y \in M$, we say that a Lipschitz curve $\gamma:[0,1] \rightarrow M$ is a geodesic between $x$ and $y$ if $\gamma$ is a minimizing curve in (2.5). Usually, geodesics are parametrized proportionally to arclength, i.e. $d(\sigma(t), \sigma(s))=v|t-s|$, where $v$ is the constant speed. We say that the metric space $(M, d)$ admits a measurable choice of 
geodesics if there exists a measurable function $f: M \times M \times[0,1] \rightarrow M$ such that the map $t \mapsto f(x, y, t)$ is a geodesic between $x$ and $y$. If for instance $M$ is a Banach space, the function

$$
f(x, y, t)=x+t(y-x)
$$

is a measurable choice of geodesics. Actually, $f$ is a continuous function. This is also true if $M$ is a compact Riemannian manifold replacing the segments with geodesics. Observe that all this notions are inherited by $\mathcal{P}_{p}(M)$ as stated in the following (see [2, 29, 20])

Proposition 2.10. If $M$ admits a measurable choice of geodesics, then for every $1 \leq p$ the space $\mathcal{P}_{p}(M)$ is complete, separable and geodesic.

The following proposition collects some important inequalities for the Wasserstein distances.

Proposition 2.11. Let $1 \leq p \leq q$. Then

1) $W_{p} \leq W_{q}$.

2) $W_{q} \leq W_{p}^{\frac{p}{q}} \operatorname{diam}(M)^{1-\frac{p}{q}}$.

Proof. Claim 1) is a direct application of Hölder's inequality. To check claim 2) let $\gamma$ be an optimal plan between its marginal $\mu, v$ for $W_{p}$. Hence

$$
\begin{aligned}
W_{q}(\mu, v) & \leq\left(\int_{M \times M} d(x, y)^{q} d \gamma\right)^{\frac{1}{q}}=\left(\int_{M \times M} d(x, y)^{p} d(x, y)^{q-p} d \gamma\right)^{\frac{1}{q}} \leq \\
& \leq W_{p}(\mu, v)^{\frac{p}{q}} \operatorname{diam}(M)^{\frac{q-p}{q}}=W_{p}(\mu, v)^{\frac{p}{q}} \operatorname{diam}(M)^{1-\frac{p}{q}} .
\end{aligned}
$$

Therefore, on bounded metric spaces all the Wasserstein distances produce the same topology. The following convexity property is an important feature of the Wasserstein distances.

Proposition 2.12. Let $p \geq 1$ and $\mu_{1}, \mu_{2}, v_{1}, v_{2} \in \mathcal{P}_{p}(M)$. Then $\forall \lambda \in[0,1]:$

$$
W_{p}\left(\lambda \mu_{1}+(1-\lambda) \mu_{2}, \lambda v_{1}+(1-\lambda) v_{2}\right)^{p} \leq \lambda W_{p}\left(\mu_{1}, v_{1}\right)^{p}+(1-\lambda) W_{p}\left(\mu_{2}, v_{2}\right)^{p} .
$$

Proof. Let $\gamma_{i}$ be an optimal plan between $\mu_{i}$ and $v_{i}$ for $i=1,2$. Observe 
that $\lambda \gamma_{1}+(1-\lambda) \gamma_{2}$ is a transport plan between $\lambda \mu_{1}+(1-\lambda) \mu_{2}$ and $\lambda v_{1}+(1-\lambda) v_{2}$. Then

$$
W_{p}\left(\lambda \mu_{1}+(1-\lambda) \mu_{2}, \lambda v_{1}+(1-\lambda) v_{2}\right)^{p} \leq \lambda W_{p}\left(\mu_{1}, v_{1}\right)^{p}+(1-\lambda) W_{p}\left(\mu_{2}, v_{2}\right)^{p} .
$$

Convexity properties of the distance are related to important geometric notions, in particular to curvature properties, see [2, 20, 29].

\section{Wasserstein spaces $\mathcal{P}_{p}(M)$ with trivial homology.}

Fixed $T \in \mathcal{I}_{1}(M)$ without boundary, $\partial T=0$, the generalized Plateau problem asks to minimize $M(S)$ among all $S \in \mathcal{I}_{2}(M)$ such that $\partial S=T$. Of course, this problem is meaningful for metric space $M$ provided the class of admissible currents is not empty and sufficiently rich. Therefore, the usual setting is to consider metric space of euclidean type, namely such that every closed 1-current is the boundary of a 2-current. In other words, by defining two metric 1-currents $T, S$ to be homologous if there exist a metric 2-current $N$ such that $T-S=\partial N$, this corresponds to spaces with trivial metric homology. By a cone construction, in [3] it is shown that Banach spaces have such a trivial homology. By a slightly modified construction, it is possible to show that more general metric spaces enjoy trivial metric homology (see [33]). Among these spaces we mention Hadamard spaces, i.e. complete simply-connected metric spaces of non-positive curvature in the Alexandrov sense, and Busemann spaces, i.e. with convex metric. We refer to $[23,24,25,28,29,30,33]$ for precise definitions and main properties. Here we focus our attention on Wasserstein spaces. As a first glance we have that the homology on $\mathcal{P}_{p}(M)$ cannot be recovered in general by homotopy. Indeed, we have the following

Proposition 3.1. $\quad \mathcal{P}_{p}(M)$ is contractible and locally contractible.

Proof. Let $\mu_{t}$ a continuous curve in $\mathcal{P}_{p}(M)$. Consider the function

$$
F:[0,1] \times[0,1] \rightarrow \mathcal{P}_{p}(M),
$$

defined by

$$
F(t, s)=s \mu_{0}+(1-s) \mu_{t}
$$


We have that $F(t, 0)=\mu_{t}$, while $F(t, 1)=\mu_{0}$. By Proposition 2.12 we have

$$
\begin{gathered}
W_{p}\left(F(t, s), F\left(t_{0}, s_{0}\right)\right)=W_{p}\left(s \mu_{0}+(1-s) \mu_{t}, s_{0} \mu_{0}+\left(1-s_{0}\right) \mu_{t_{0}}\right) \leq \\
\leq W_{p}\left(s \mu_{0}+(1-s) \mu_{t}, s \mu_{0}+(1-s) \mu_{t_{0}}\right)+ \\
+W_{p}\left(s \mu_{0}+(1-s) \mu_{t_{0}}, s_{0} \mu_{0}+\left(1-s_{0}\right) \mu_{t_{0}}\right) \leq \\
\leq(1-s)^{\frac{1}{p}} W_{p}\left(\mu_{t}, \mu_{t_{0}}\right)+W_{p}\left(s \mu_{0}+(1-s) \mu_{t_{0}}, s_{0} \mu_{0}+\left(1-s_{0}\right) \mu_{t_{0}}\right) \leq \varepsilon
\end{gathered}
$$

provided that $\left|s-s_{0}\right|,\left|t-t_{0}\right| \leq \delta$ because of the continuity of $\mu_{t}$. To check the second claim consider the ball $B(v, 1) \subset \mathcal{P}_{p}(M)$. If $\mu_{t}$ is a curve on the ball such that $\mu_{0}=v$, by Proposition 2.12 and (3.1) we have

$$
W_{p}(v, F(s, t))=W_{p}\left(\mu_{0}, s \mu_{0}+(1-s) \mu_{t}\right) \leq(1-s)^{\frac{1}{p}} W_{p}\left(\mu_{0}, \mu_{t}\right) \leq 1 .
$$

Observe that by defining the metric homology through metric currents, it results that the homology is not a topological invariant. In particular, in this metric setting, at least Lipschitz regularity of maps between metric spaces is needed. Therefore, although Wasserstein spaces are contractible, in general it could happen that this contraction is not a Lipschitz map. Therefore, Wasserstein spaces could have nontrivial metric homology.

\section{1 - Barycenter maps.}

A possible strategy to investigate the homology of $\mathcal{P}_{p}(M)$ is to relate the geometry of $\mathcal{P}_{p}(M)$ with the geometry of the underlying space $M$. This program can be certainly realized if a barycenter map is available. Here for barycenter map we mean a function

$$
\beta: \mathcal{P}_{1}(M) \rightarrow M,
$$

which satisfy the conditions:

(1) $\forall x \in M: \beta\left(\delta_{x}\right)=x$,

(2) $\beta \in \operatorname{Lip}_{1}\left(\mathcal{P}_{1}(M), M\right)$.

Because of Proposition $2.11 \beta \in \operatorname{Lip}_{1}\left(\mathcal{P}_{p}(M), M\right)$ for every $p \geq 1$ as well.

However, we point out that the existence of barycenter maps produces some restrictions on the geometry of the space $M$. 
- If $x, y \in M$ we can define the curve $\sigma(t):=\beta\left((1-t) \delta_{x}+t \delta_{y}\right)$ which turns out to be a geodesic curve. Furthermore the distance is convex along such geodesics. Hence, if geodesics on $M$ are unique then $M$ is a Busemann space.

- If $M$ is a Riemannian manifold then $M$ must have sectional curvature $\leq 0$. Indeed, if $M_{0} \subset M$ it also admits a barycenter map. Choosing $M_{0}$ sufficiently small, geodesics on $M_{0}$ are unique and then the distance is convex on $M_{0}$. This implies that $M$ has sectional curvature $\leq 0$.

Actually, barycenter maps can be canonically constructed on Hadamard spaces (see [20, 24, 29]). Consider a Lipschitz cycle $\gamma:[a, b] \rightarrow M$ on the Hadamard space $M$. It results that also the curve $\delta_{\gamma(t)}$ on the Wasserstein space $\mathcal{P}_{p}(M)$ is a Lipschitz curve. Therefore, this curve corresponds to a metric 1-current on $\mathcal{P}_{p}(M)$, which we denote by $T_{\gamma}$, defined as

$$
T_{\gamma}\left(f_{0}, f_{1}\right)=\int_{a}^{b} f_{0}\left(\delta_{\gamma(t)}\right) \frac{d}{d t} f_{1}\left(\delta_{\gamma(t)}\right) d t .
$$

We have the following

THEOREM 3.2. Let M be an Hadamard space. Then for every Lipschitz cycle $\gamma$ on $M$ the 1-currents $T_{\gamma}$ is homologous to zero in $\mathcal{P}_{p}(M)$.

Proof. We have $T_{\gamma} \in \mathcal{I}_{1}^{c}\left(\mathcal{P}_{p}(M)\right)$. Therefore, $\beta_{\#} T_{\gamma}$ is a closed metric current on $M$. By the cone construction of [33] there exists $S \in \mathcal{I}_{2}(M)$ such that $\beta_{\#} T_{\gamma}=\partial S$. Consider the canonical embedding $i: M \rightarrow \mathcal{P}_{p}(M)$ defined by $i(x)=\delta_{x}$. Since $i \circ \beta\left(\delta_{x}\right)=\delta_{x}$ for every $x \in M$, we have

$$
T_{\gamma}=i_{\#}\left(\beta_{\#}\left(T_{\gamma}\right)\right)=i_{\#}(\partial S)=\partial\left(i_{\#} S\right) .
$$

Hence, by Theorem 3.2 the 1-currents $T_{\gamma}$ belong to the same homology class of $\mathcal{P}_{p}(M)$. However, we don't know whether there could exist other homology classes in $\mathcal{P}_{p}(M)$ or not. In [16] is discussed an approach by differential forms on $\mathcal{P}_{2}\left(\mathbb{R}^{d}\right)$, showing that in some sense the space $\mathcal{P}_{2}\left(\mathbb{R}^{d}\right)$ has trivial homology. We may conjecture that actually also $H_{1}\left(\mathcal{P}_{p}(M)\right)=\{0\}$ for Hadamard spaces $M$. Probably, it would be necessary different tools to prove or reject this conjecture. In the next sections we develop a more general approach, based on mass transportation tools, ables to check if two metric closed 1-currents are homologous in $\mathcal{P}_{p}(M)$. By 
using these results we will prove that $\mathcal{P}_{p}\left(T^{n}\right)$ is an example of Wasserstein space with distinct homology classes in a metric sense. Since every complete simply-connected Riemannian manifolds of non-positive sectional curvature is an Hadamard space, to recover non trivial homology on $\mathcal{P}_{p}(M)$ we have to look for manifolds with local sectional curvature $\leq 0$ or with non-negative curvature. Observe that there is no canonical way to define barycenter maps on such spaces. Furthermore, whenever the space $M$ is non-contractible, such a barycenter map cannot exist.

THEOREM 3.3. Every non-contractible metric space $(M, d)$ does not admit a barycenter map.

Proof. Suppose by contradiction that there exists a barycenter map $\beta: \mathcal{P}_{p}(M) \rightarrow M$. Consider a closed continuous curve $\gamma:[0,1] \rightarrow M$ not homotopic to a constant. Let $i: M \rightarrow \mathcal{P}_{p}(M)$ be the canonical isometry defined by $i(x)=\delta_{x}$. Take the continuous function $F:[0,1] \times$ $\times[0,1] \rightarrow \mathcal{P}_{p}(M)$ defined by (3.1) with $\mu_{t}=i(\gamma(t))$ and consider the continuous composition $\beta \circ F:[0,1] \times[0,1] \rightarrow M$. We have

$$
\begin{gathered}
\beta \circ F(t, 0)=\beta(i(\gamma(t))=\gamma(t), \\
\beta \circ F(t, 1)=\beta\left(\mu_{0}\right),
\end{gathered}
$$

which is a contradiction since the curve $\gamma$ is not homotopic to a constant.

Since we can always define barycenters on the set $\Delta$ of Dirac deltas by $\beta\left(\delta_{x}\right)=x$, certainly satisfying the Lipschitz condition of barycenters, a possible strategy is to try to extend the map $\beta$ to the whole space $\mathcal{P}_{1}(M)$ in a Lipschitz way. The following statement, adapted to the case of curvature $\leq 0$ and $\geq 0$ in the Alexandorov sense, is taken from [25].

Theorem 3.4 (General Kirszbraun theorem). Let E, F geodesics metric spaces such that $E$ has curvature $\geq 0$, while $F$ is complete with global curvature $\leq 0$. If $A \subset E$, every $f \in \operatorname{Lip}(A, F)$ admits a Lipschitz extension with the same Lipschitz constant.

We remark that this statement actually holds whenever the curvatures are bounded by a constant $k \in \mathbb{R}$ instead of 0 , provided that $\operatorname{diam}(f(A)) \leq \frac{1}{2} D_{k}$, where $D_{k}=\frac{\pi}{\sqrt{k}}$ if $k>0, D_{k}=+\infty$ otherwise. The following example shows that in Theorem 3.4 the assumption of target space of global curvature $\leq 0$ cannot be reduced to a local one. 
ExAmple 3.5. Consider the flat torus " $T{ }^{n}$. It is known that the space $\left(\mathcal{P}\left(T^{n}\right), W_{2}\right)$ has curvature $\geq 0$ (see $[2,20,29]$ ). Of course, "T $T^{n}$ is noncontractible and it has local curvature $\leq 0$. Therefore, by Theorem 3.3 we infer that the 1-Lipschitz map

$$
\beta: \Delta \rightarrow T^{n}
$$

defined by $\beta\left(\delta_{x}\right)=x$ cannot be extended in a Lipschitz way to the whole $\mathcal{P}\left(T^{n}\right)$.

If $M$ is a manifold the same example can be done using the isometric embedding of $M$ in $\mathbb{R}^{k}$ for $k$ big enough.

\section{Entire metric currents on $\mathcal{P}_{p}(M)$.}

On a general manifold $M$ we need to base our approach on more intrinsic instruments. In [34] is developed a general approach to define an homology for the class of metric spaces which are locally contractible in a Lipschitz way. Among them we mention metric space with global Alexandrov curvature bounded from above. However, Wasserstein spaces does not enjoy these curvature properties, unless for $\mathcal{P}_{p}(\mathbb{R})$, see [21]. Here we start to study some relationships between the entire homology of $\mathcal{P}_{p}(M)$ and the real homology of $M$. The basic idea is to relate these homologies by the continuity equation (1.2).

We begin by recalling how a Lipschitz curve $\rho:[a, b] \rightarrow \mathcal{P}_{p}(M)$ is identified with an entire 1-current on $\mathcal{P}_{p}(M)$ which we denote by $T_{\rho}$. Following [3], $T_{\rho}$ is defined by

$$
T_{\rho}\left(f_{0}, f_{1}\right)=\int_{a}^{b} f_{0}(\rho(t)) \frac{d}{d t} f_{1}(\rho(t)) d t .
$$

If we have to take in account a multiplicity function $\theta \in L^{1}$, we define

$$
T_{\theta, \rho}\left(f_{0}, f_{1}\right)=\int_{a}^{b} \theta(t) f_{0}(\rho(t)) \frac{d}{d t} f_{1}(\rho(t)) d t .
$$

For reader convenience, in the following lemma we compute the total mass of $T_{\theta, \rho}$.

Lemma 4.1. For every $\theta \in L^{1}(\mathbb{R}, Z)$ it results $T_{\theta, \rho} \in \mathcal{I}_{1}\left(\mathcal{P}_{p}(M)\right)$, and its 
total mass is given by

$$
M\left(T_{\theta, \rho}\right)=\int_{a}^{b}|\theta(t)||\dot{\rho}|_{p}(t) d t
$$

where $|\dot{\rho}|_{p}(t)$ is the metric derivative of $\rho$ w.r.t. $W_{p}$.

Proof. Notice that $T_{\theta, \rho}=\rho_{\#}\left(T_{\theta}\right)$, so that $T_{\theta, \rho} \in \mathcal{I}_{1}\left(\mathcal{P}_{p}(M)\right)$. Since $\frac{d}{d t} f_{1}(\rho(t)) \leq \operatorname{Lip}\left(f_{1}\right)|\dot{\rho}|_{p}(t)$ we easily get

$$
T_{\theta, \rho}\left(f_{0}, f_{1}\right) \leq \operatorname{Lip}\left(f_{1}\right) \int_{a}^{b}|\theta(t)|\left|f_{0}(\rho(t)) \| \dot{\rho}\right|_{p}(t) d t .
$$

Hence, for any Borel partition $\left\{B_{j}\right\}_{j}$ of $\mathcal{P}_{p}(M)$, and 1-Lipschitz functions $f_{1}^{j}$ we have $\sum_{j=1}^{N} T_{\theta, \rho}\left(\chi_{B_{j}}, f_{1}^{j}\right) \leq \int_{a}^{b}|\theta(t)||\dot{\rho}|_{p}(t) d t$. Therefore we get

$$
M\left(T_{\theta, \rho}\right) \leq \int_{a}^{b}|\theta(t)||\dot{\rho}|_{p}(t) d t
$$

To prove the opposite inequality, let us start with the case $\theta=\chi_{I}$ for a given interval $I$. Now let $\varepsilon>0$ be fixed. Since $\int_{I}|\dot{\rho}|_{p}(t) d t$ coincides with the length of the curve $\rho$ in $I$ (see [4]), there is a partition $\left\{t_{1}, \ldots, t_{N+1}\right\}$ of $I$ such that

$$
\int_{I}|\dot{\rho}|_{p}(t) d t \leq \sum_{j=1}^{N} W_{p}\left(\rho\left(t_{j+1}\right), \rho\left(t_{j}\right)\right)+\varepsilon .
$$

For any $j=1, \ldots, N+1$, consider $f_{1}^{j}(\mu):=W_{p}\left(\rho\left(t_{j}\right), \mu\right) ; f_{1}^{j}$ are 1-Lipschitz functions and

$$
W_{p}\left(\rho\left(t_{j+1}\right), \rho\left(t_{j}\right)\right)=\int_{t_{j}}^{t_{j+1}} \frac{d}{d s} f_{1}^{j}(\rho(s)) d s .
$$

Defining $B_{j}:=\rho\left(\left[t_{j}, t_{j+1}\right]\right)$ for $j=1, \ldots, N$ and $B_{N+1}:=\mathcal{P}_{p}(M) \backslash \cup_{j=1}^{N} B_{j}$, it follows that

$$
\int_{I}|\dot{\rho}|_{p}(t) d t \leq \sum_{j=1}^{N} \int_{t_{j}}^{t_{j+1}} \frac{d}{d s} f_{1}^{j}(\rho(s)) d s+\varepsilon=\sum_{j=1}^{N+1} T_{\rho}\left(\chi_{B_{j}}, f_{1}^{j}\right)+\varepsilon .
$$


Being $\left\{B_{j}\right\}_{j}$ a Borel partition of $\mathcal{P}_{p}(M)$, taking into account remark 2.2 and letting $\varepsilon \rightarrow 0$, we get

$$
\int_{I}|\dot{\rho}|_{p}(t) d t \leq M\left(T_{\rho}\llcorner\rho(I)) .\right.
$$

Therefore, if a Lipschitz curve $\rho$ is defined on the interval $J$, we have proved that

$$
M\left(T_{\rho}\right)=\int_{J}|\dot{\rho}|_{p}(t) d t .
$$

In the general case we approximate $\theta$ by piece-wise constant functions. Therefore, there exists a Borel partition of $[a, b]$ made of intervals $J_{i}$ and constants $c_{i}$ such that

$$
\int_{a}^{b}|\theta(t)||\dot{\rho}|_{p}(t) d t=\sum_{i=1}^{+\infty} c_{i} \int_{J_{i}}|\dot{\rho}|_{p}(t) d t=\sum_{i=1}^{+\infty} M\left(T_{\theta, \rho}\left\llcorner\rho\left(J_{i}\right)\right)=M\left(T_{\theta, \rho}\right) .\right.
$$

Formula (4.1) also holds true for any $\theta \in L^{1}$ and for any metric space $E$ instead of $\mathcal{P}_{p}(M)$. An immediate consequence of the continuity equation is the following

Lemma 4.2. For every solution $(\rho, v)$ of the continuity equation (1.2) and for every $f \in \mathcal{C}^{1}(M)$ it results

$$
\frac{d}{d t}\left(\int_{M} f(x) d \rho_{t}\right)=\int_{M}\left\langle d_{x} f(x), v(x, t)\right\rangle d \rho_{t}
$$

in the sense of distributions.

Proof. For every $\varphi \in \mathcal{C}_{c}^{\infty}(0,1)$ we evaluate

$$
\begin{aligned}
\int_{0}^{1} \frac{d}{d t}\left(\int_{M} f(x) d \rho_{t}\right) \varphi(t) d t & =-\int_{0}^{1} \dot{\varphi}(t)\left(\int_{M} f(x) d \rho_{t}\right) d t= \\
& -\int_{0}^{1}\left(\int_{M} \dot{\varphi}(t) f(x) d \rho_{t}\right) d t=\frac{\partial \rho}{\partial t}(\varphi f)=-\nabla \cdot(v \rho)(\varphi f)= \\
= & \int_{0}^{1}\left(\int_{M}\left\langle d_{x} f(x), v\right\rangle d \rho_{t}\right) \varphi(t) d t .
\end{aligned}
$$


Actually, it turns out that the map $f \mapsto \int_{M} f d \rho_{t} \in W_{l o c}^{1,1}(0,1)$. Therefore, formula (4.2) holds for a.e. $t \in(0,1)$.

We define a natural operator which associates to each entire metric 1-current $T \in \boldsymbol{I}_{1}\left(\mathcal{P}_{p}(M)\right)$ a normal euclidean 1-current $\widetilde{T} \in \mathcal{N}_{1}(M)$. The structure Theorem 2.4 gives a representation for $T=\sum_{i=1}^{\infty} T_{\theta_{i}, \rho_{i}}$, and to each $\rho_{i}$ is associated its tangent vector $v_{i}$. Then for a 1 -form $\omega$ on $M$ we can define

$$
\widetilde{T}(\omega)=\sum_{i=1}^{\infty} \int_{a_{i}}^{b_{i}} \theta_{i}(t)\left(\int_{M}\left\langle\omega(x), v_{i}(t, x)\right\rangle d \rho_{i}\right) d t .
$$

We get the following

Proposition 4.3. For every $T \in \boldsymbol{I}_{1}\left(\mathcal{P}_{p}(M)\right)$ it results $\widetilde{T} \in \mathcal{N}_{1}(M)$.

Proof. To compute the mass we evaluate

$$
|\widetilde{T}(\omega)| \leq\|\omega\|_{\infty} \sum_{i=1}^{\infty} \int_{a_{i}}^{b_{i}}\left|\theta_{i}\right|\left(\int_{M}\left|v_{i}(t, x)\right| d \rho_{i}\right) d t \leq\|\omega\|_{\infty} \sum_{i=1}^{\infty} \int_{a_{i}}^{b_{i}}\left|\theta_{i}\right|\left\|v_{i}\right\|_{L^{p}\left(\rho_{i}\right)} d t .
$$

Using Lemma 4.1, Theorem 2.8 and Theorem 2.4 we get

$$
|\widetilde{T}(\omega)| \leq\|\omega\|_{\infty} \sum_{i=1}^{\infty} \int_{a_{i}}^{b_{i}}\left|\theta_{i}\left\|\left.\dot{\rho}_{i}\right|_{p}(t) d t=\right\| \omega\left\|_{\infty} \sum_{i=1}^{\infty} M\left(T_{\theta_{i}, \rho_{i}}\right)=\right\| \omega \|_{\infty} M(T) .\right.
$$

Taking the supremum with respect to $\|\omega\|_{\infty} \leq 1$ it results

$$
M(\widetilde{T}) \leq M(T)<+\infty .
$$

To handle with the boundary operator, we state the following preliminary remark. For every $f \in \mathcal{C}^{1}(M)$ the map $f_{0}: \mathcal{P}_{p}(M) \rightarrow \mathbb{R}$ defined by $f_{0}(\mu)=\int_{M} f d \mu$ is a bounded Lipschitz function. Indeed, recalling Proposition 2.11 and Theorem 2.6 it results

$$
\left|f_{0}(\mu)-f_{0}(v)\right|=\left|\int_{M} f d(\mu-v)\right| \leq \operatorname{Lip}(f) W_{1}(\mu, v) \leq \operatorname{Lip}(f) W_{p}(\mu, v) .
$$


Using Lemma 4.2 we evaluate

$$
\begin{aligned}
& \partial \widetilde{T}(f)=\widetilde{T}(d f)=\sum_{i=1}^{\infty} \int_{a_{i}}^{b_{i}} \theta_{i}(t)\left(\int_{M}\left\langle d f(x), v_{i}(t, x)\right\rangle d \rho_{i}\right) d t= \\
& =\sum_{i=1}^{\infty} \int_{a_{i}}^{b_{i}} \theta_{i}(t) \frac{d}{d t}\left(\int_{M} f d \rho_{i}\right) d t=\sum_{i=1}^{\infty} \int_{a_{i}}^{b_{i}} \theta_{i}(t) \frac{d}{d t} f_{0}\left(\rho_{i}(t)\right) d t=T\left(1, f_{0}\right)=\partial T\left(f_{0}\right) .
\end{aligned}
$$

Observing that $\|f\|_{\infty} \leq 1 \Rightarrow\left\|f_{0}\right\|_{\infty} \leq 1$, we get $M(\partial \widetilde{T})<+\infty$.

If multiplicity functions $\theta_{i}$, or more generally multiplicity measures $\mu_{i}$, are associated to the curves $\rho_{i}$, we will define

$$
\widetilde{T}(\omega)=\sum_{i=1}^{\infty} \int_{\mathbb{R}}\left(\int_{M}\left\langle\omega(x), v_{i}(t, x)\right\rangle d \rho_{i}\right) d \mu_{i}(t) .
$$

We will apply a similar definition for multiplicity depending on a more parameter. It is easy to define the operator $\sim$ also for entire 0-currents, indeed if $S$ is an entire 0-current then its representation is given by $S=\sum_{i=1}^{\infty} a_{i} \delta_{\mu_{i}}$ and then we define $\widetilde{S}=\sum_{i=1}^{\infty} a_{i} \mu_{i}$.

REMARK 4.4. We attract the attention of the reader to the fact that with this definition $\widetilde{T}_{\rho}$ coincides with the euclidean current $T_{\rho, v}$ involved in problem $\mathbf{P}$.

The following theorem holds

Theorem 4.5. The diagram

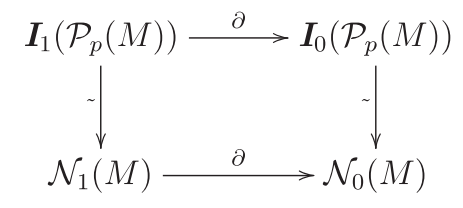

commutes.

Proof. Take $T \in \boldsymbol{I}_{1}\left(\mathcal{P}_{p}(M)\right), T=\sum_{i=1}^{\infty} T_{\theta_{i}, \rho_{i}}$ according to Theorem 2.4. If $f$ is a Lipschitz bounded function on $\mathcal{P}_{p}(M)$ we have:

$$
\partial T(f)=\sum_{i=1}^{\infty} \int_{a_{i}}^{b_{i}} \theta_{i}(t) \frac{d}{d t} f\left(\rho_{i}(t)\right) d t=\sum_{i=1}^{\infty}\left(\left[\theta_{i}(t) f\left(\rho_{i}(t)\right)\right]_{a_{i}}^{b_{i}}-\int_{a_{i}}^{b_{i}} f\left(\rho_{i}(t)\right) d \theta_{i}^{\prime}(t)\right) .
$$


For $f \in \mathcal{C}^{1}(M)$, passing to the operator we get

$$
\widetilde{\partial T}(f)=\sum_{i=1}^{\infty}\left(\int_{M} f d\left(\theta_{i}\left(b_{i}^{-}\right) \rho_{i}\left(b_{i}\right)-\theta_{i}\left(a_{i}^{+}\right) \rho_{i}\left(a_{i}\right)\right)-\int_{a_{i}}^{b_{i}}\left(\int_{M} f d \rho_{i}\right) d \theta_{i}^{\prime}(t)\right) .
$$

On the other hand, if $f \in \mathcal{C}^{1}(M)$, taking into account Lemma 4.2, it results

$$
\begin{aligned}
& \partial \widetilde{T}(f)=\widetilde{T}(d f)=\sum_{i=1}^{\infty} \int_{a_{i}}^{b_{i}} \theta_{i}(t)\left(\int_{M}\left\langle d f(x), v_{i}(t, x)\right\rangle d \rho_{i}\right) d t= \\
& =\sum_{i=1}^{\infty} \int_{a_{i}}^{b_{i}} \theta_{i}(t) \frac{d}{d t}\left(\int_{M} f(x) d \rho_{i}\right) d t= \\
& =\sum_{i=1}^{\infty}\left(\int_{M} f d\left(\theta_{i}\left(b_{i}^{-}\right) \rho_{i}\left(b_{i}\right)-\theta_{i}\left(a_{i}^{+}\right) \rho_{i}\left(a_{i}\right)\right)-\int_{a_{i}}^{b_{i}}\left(\int_{M} f d \rho_{i}\right) d \theta_{i}^{\prime}(t)\right)=\widetilde{\partial T}(f) .
\end{aligned}
$$

A fundamental step toward the proof of our initial claim would be to answer the following question: let $\rho$ and $\bar{\rho}$ be two closed Lipschitz curves in $\mathcal{P}_{p}(M)$ such that $T_{\rho}$ and $T_{\bar{\rho}}$ lie in the same homology class of $H_{1}\left(\mathcal{P}_{p}(M)\right)$, is it true that $\widetilde{T}_{\rho}$ and $\widetilde{T}_{\bar{\rho}}$ are in the same real homology class of $H_{1}(M, \mathbb{R})$ ?

This would be accomplished by defining a suitable ${ }^{\sim}$ operator for 2 currents and showing that also the diagram

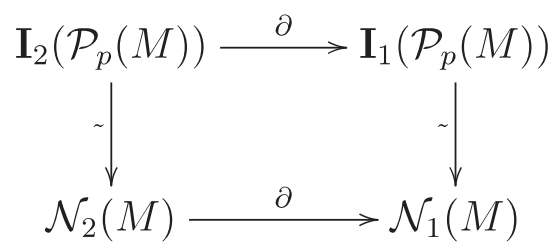

commutes. Unfortunately we are currently not able to prove this last fact in full generality. There are two main obstacles, one is the absence of a natural analogous of the continuity equation in higher dimensions, the other is that in the representation Theorem 2.4 for entire 2-currents it is not known if one can choose regular sets $K_{i}$. 


\section{Metric currents and metric homology of $\mathcal{P}_{p}\left(T^{n}\right)$.}

5.1 - The $~$ operator for 2-currents on $\mathcal{P}_{p}(M)$.

As stated in the Introduction, for every Mather's minimal measure $\mu$ in a given homology class there are minimal Eulerian representations $(\rho, v)$, i.e. solutions of problem $\mathbf{P}$ (see [11] and [9] for details and related results). It is natural to ask if each of them enjoys a certain intrinsic minimality, namely that it minimizes an action functional (in its entire homology class in $H_{1}\left(\mathcal{P}_{p}(M)\right)$. In order to discuss this question, in this Section we define a $\sim$ operator for metric 2-currents on $\mathcal{P}_{p}(M)$. In the next Sections we will focus our attention on the flat n-torus ' ${ }^{n}$ on which some of the program can be carried on and it is easier to show which are the obstacles in doing it in full generality. This will also help to understand the geometric structure of $\mathcal{P}_{p}(M)$. Let us recall from [3] that if $N \in \boldsymbol{I}_{2}\left(\mathcal{P}_{p}(M)\right)$ we have

(5.1) $N\left(f_{0}, f_{1}, f_{2}\right)=$

$$
=\sum_{i=0}^{+\infty} \int_{K_{i}} \theta_{i}(s, t) f_{0}\left(\varphi_{i}(s, t)\right) \operatorname{det}\left(\begin{array}{ll}
\partial_{s} f_{1}\left(\varphi_{i}(s, t)\right), & \partial_{t} f_{1}\left(\varphi_{i}(s, t)\right) \\
\partial_{s} f_{2}\left(\varphi_{i}(s, t)\right), & \partial_{t} f_{2}\left(\varphi_{i}(s, t)\right)
\end{array}\right) d s d t
$$

where $\varphi_{i}: K_{i} \rightarrow \mathcal{P}_{p}(M)$ are bi-Lipschitz curves. To consider tangent vectors we give the following

DeFInition 5.1. We say that $\varphi_{i}$ is regular if $K_{i}=\left[a_{i}, b_{i}\right] \times\left[c_{i}, d_{i}\right]$ is a rectangle of $\mathbb{R}^{2}$ and the tangent vectors $v_{1}(x, s, t), v_{2}(x, s, t)$, obtained by Theorem 2.8 fixing respectively the third and the second variable of $\varphi_{i}$, are well defined Borel vector fields. If the curves $\varphi_{i}$ in (5.1) are regular for every $i$ we say that $N \in \boldsymbol{I}_{2}^{\text {reg }}\left(\mathcal{P}_{p}(M)\right)$.

For $N \in \boldsymbol{I}_{2}^{\text {reg }}\left(\mathcal{P}_{p}(M)\right)$ we can formally define

(5.2) $\tilde{N}(d \omega):=$

$$
=-\sum_{i=0}^{+\infty} \int_{K_{i}} \theta_{i}(s, t)\left[\frac{\partial}{\partial t} \int_{M}\left\langle\omega, v_{1}\right\rangle d \varphi_{i}(s, t)-\frac{\partial}{\partial s} \int_{M}\left\langle\omega, v_{2}\right\rangle d \varphi_{i}(s, t)\right] d s d t .
$$

Observe that the right hand side of (5.2) formally correspond to a closed 
1-current. Indeed, for every $f \in \mathcal{C}^{1}(M)$, by Lemma 4.2 it results

$$
\begin{aligned}
& \frac{\partial}{\partial t} \int_{M}\left\langle d f, v_{1}\right\rangle d \varphi_{i}(s, t)= \\
&=\frac{\partial^{2}}{\partial t \partial s} \int_{M} f d \varphi_{i}(s, t)=\frac{\partial^{2}}{\partial s \partial t} \int_{M} f d \varphi_{i}(s, t)=\frac{\partial}{\partial s} \int_{M}\left\langle d f, v_{2}\right\rangle d \varphi_{i}(s, t)
\end{aligned}
$$

in the sense of distribution. To relate $N \in \boldsymbol{I}_{2}^{\text {reg }}\left(\mathcal{P}_{p}(M)\right)$ to a well defined euclidean current $\tilde{N}$, we need some more regularity of the curves $\varphi_{i}$. Therefore, for $\varepsilon>0$ and denoting for simplicity $u:=(s, t)$, we consider the following convolution approximation of a Lipschitz curve $\varphi: \mathbb{R}^{2} \rightarrow \mathcal{P}_{p}(M)$

$$
\begin{gathered}
\varphi^{\varepsilon}(u)=\int_{\mathbb{R}^{2}} \rho_{\varepsilon}(u-v) \varphi(v) d v \in \mathcal{P}(M), \\
\left\langle E_{i}^{\varepsilon}(u), \omega\right\rangle=\int_{\mathbb{R}^{2}} \rho_{\varepsilon}(u-v)\left(\int_{M}\left\langle\omega, v_{i}\right\rangle d \varphi(v)\right) d v \quad i=1,2
\end{gathered}
$$

where $\rho_{\varepsilon}$ is a standard convolution kernel. Observing that

$$
\left\langle E_{i}^{\varepsilon}(u), \omega\right\rangle=\int_{\mathbb{R}^{2}}\left(\int_{M} \rho_{\varepsilon}(u-v)\left\langle\omega, v_{i}\right\rangle d \varphi(v)\right) d v=\int_{M}\left\langle\omega, v_{i}\right\rangle d \varphi^{\varepsilon}(u),
$$

it follows that the tangent vectors correspondent to $\varphi^{\varepsilon}$ are $v_{i}, i=1,2$, as well. We have the following

LEMMA 5.2. Under the previous assumptions we have

(1) $\lim _{\varepsilon \rightarrow 0^{+}} \varphi^{\varepsilon}=\varphi$ uniformly with respect to the Wasserstein metric,

(2) The functions $\varphi^{\varepsilon}$ are equi-Lipschitz,

(3) $E_{1}^{\varepsilon}$ and $E_{2}^{\varepsilon}$ solve the continuity equations

$$
\partial_{s} \varphi^{\varepsilon}+\nabla \cdot E_{1}^{\varepsilon}=0, \quad \partial_{t} \varphi^{\varepsilon}+\nabla \cdot E_{2}^{\varepsilon}=0,
$$

(4) $\lim _{u \rightarrow \bar{u}} E_{i}^{\varepsilon}(u)=E_{i}^{\varepsilon}(\bar{u})$ weakly for $i=1,2$.

Proof. 1) We use Kantorovich duality. If $p=1$ consider $f \in \operatorname{Lip}_{1}(M)$. We evaluate

$$
\int_{M} f d\left(\varphi^{\varepsilon}(u)-\varphi(u)\right)=\int_{M} f d\left(\int_{\mathbb{R}^{2}} \rho_{\varepsilon}(u-v)[\varphi(v)-\varphi(u)] d v\right)=
$$




$$
\begin{gathered}
=\int_{\mathbb{R}^{2}}\left(\rho_{\varepsilon}(u-v) \int_{M} f d(\varphi(v)-\varphi(u))\right) d v \leq \int_{\mathbb{R}^{2}} \rho_{\varepsilon}(u-v) W_{1}(\varphi(v), \varphi(u)) d v \leq \\
\leq \operatorname{Lip}(\varphi) \int_{\mathbb{R}^{2}} \rho_{\varepsilon}(u-v)\|u-v\| d v \leq \operatorname{Lip}(\varphi) \varepsilon .
\end{gathered}
$$

Taking the supremum with respect to $f \in \operatorname{Lip}_{1}(M)$ the claim follows. If $p>1$, take a couple $(f, g) \in L^{1}\left(\varphi^{\varepsilon}(u)\right) \times L^{1}(\varphi(u))$ such that $f(x)+g(y) \leq$ $\leq d(x, y)^{p}$ for every $(x, y) \in M$. Then we compute

$$
\begin{aligned}
& \int_{M} f d \varphi^{\varepsilon}(u)+\int_{M} g d \varphi(u)=\int_{\mathbb{R}^{2}} \rho_{\varepsilon}(u-v)\left(\int_{M} f d \varphi(v)\right) d v+ \\
& \int_{\mathbb{R}^{2}} \rho_{\varepsilon}(u-v)\left(\int_{M} g d \varphi(u)\right) d v=\int_{\mathbb{R}^{2}} \rho_{\varepsilon}(u-v)\left(\int_{M} f d \varphi(v)+\int_{M} g d \varphi(u)\right) d v \leq \\
& \leq \int_{\mathbb{R}^{2}} \rho_{\varepsilon}(u-v) W_{p}(\varphi(v), \varphi(u)) d v \leq \operatorname{Lip}(\varphi) \int_{\mathbb{R}^{2}} \rho_{\varepsilon}(u-v)\|u-v\| d v \leq \operatorname{Lip}(\varphi) \varepsilon .
\end{aligned}
$$

Taking the supremum with respect to $(f, g)$ the claim follows.

2) For $p=1$ let $f \in \operatorname{Lip}_{1}(M)$. We compute

$$
\begin{aligned}
& \int_{M} f d\left(\varphi^{\varepsilon}(u)-\varphi^{\varepsilon}(\bar{u})\right)=\int_{\mathbb{R}^{2}} \rho_{\varepsilon}(v)\left(\int_{M} f d(\varphi(u-v)-\varphi(\bar{u}-v))\right) d v \leq \\
& \leq W_{1}(\varphi(u-v), \varphi(\bar{u}-v)) \leq \operatorname{Lip}(\varphi)\|u-\bar{u}\| .
\end{aligned}
$$

Taking the supremum with respect to $f \in \operatorname{Lip}_{1}(M)$ we obtain

$$
W_{1}\left(\varphi^{\varepsilon}(u), \varphi^{\varepsilon}(\bar{u})\right) \leq \operatorname{Lip}(\varphi)\|u-\bar{u}\| .
$$

A similar argument works for $p>1$.

3) $\partial_{s} \varphi^{\varepsilon}+\nabla \cdot E_{1}^{\varepsilon}=\int_{\mathbb{R}^{2}} \rho_{\varepsilon}(u-v)\left(\partial_{s} \varphi+\nabla \cdot\left(v_{1} \varphi\right)\right) d v=0$ since $\left(\varphi, v_{1}\right)$ solves the continuity equation with respect to the $s$ variable. The same argument works for the other equation as well. 
4) For every 1-form $\omega$ we have

$$
\begin{aligned}
\left\langle E_{i}^{\varepsilon}(u)-E_{i}^{\varepsilon}(\bar{u}), \omega\right\rangle=\int_{\mathbb{R}^{2}} \rho_{\varepsilon}(u-v)\left(\int_{M}\left\langle\omega, v_{i}\right\rangle d \varphi(v)\right) d v- & \\
& -\int_{\mathbb{R}^{2}} \rho_{\varepsilon}(\bar{u}-v)\left(\int_{M}\left\langle\omega, v_{i}\right\rangle d \varphi(v)\right) d v= \\
& =\int_{\mathbb{R}^{2}}\left(\int_{M}\left\langle\omega, v_{i}\right\rangle d \varphi(v)\right)\left[\rho_{\varepsilon}(u-v)-\rho_{\varepsilon}(\bar{u}-v)\right] d v .
\end{aligned}
$$

Therefore, by Theorem 2.8 we estimate

$$
\begin{aligned}
\left|\left\langle E_{i}^{\varepsilon}(u)-E_{i}^{\varepsilon}(\bar{u}), \omega\right\rangle\right| & \leq\|\omega\|_{\infty} \int_{\mathbb{R}^{2}}\left|\partial_{i} \varphi\right|_{p}\left|\rho_{\varepsilon}(u-v)-\rho_{\varepsilon}(\bar{u}-v)\right| d v \leq \\
& \leq C_{1} \operatorname{Lip}(\varphi)\|\omega\|_{\infty} \frac{1}{\varepsilon^{3}} \int_{B(u, \varepsilon) \cup B(\bar{u}, \varepsilon)}\|u-\bar{u}\| d v \leq \frac{C_{2}}{\varepsilon}\|u-\bar{u}\| .
\end{aligned}
$$

In particular, the last computation of the above proof shows that the function $\left\langle E_{i}^{\varepsilon}(u), \omega\right\rangle$ is Lipschitz with respect to the $u$ variable and then the formula (5.2) for $\varphi_{i}^{\varepsilon}$ in place of $\varphi_{i}$ is well defined. Therefore, for $N \in \boldsymbol{I}_{2}^{\text {reg }}\left(\mathcal{P}_{p}(M)\right)$ we define

$$
\begin{aligned}
& \text { (5.3) } \tilde{N}(d \omega):= \\
& =-\sum_{i=0}^{+\infty} \lim _{\varepsilon \rightarrow 0^{+}} \int_{K_{i}} \theta_{i}(s, t)\left[\frac{\partial}{\partial t} \int_{M}\left\langle\omega, v_{1, i}\right\rangle d \varphi_{i}^{\varepsilon}(s, t)-\frac{\partial}{\partial s} \int_{M}\left\langle\omega, v_{2, i}\right\rangle d \varphi_{i}^{\varepsilon}(s, t)\right] d s d t,
\end{aligned}
$$

where $v_{k, i}, k=1,2$, are the tangent vectors associated to the curves $\varphi_{i}^{\varepsilon}$. By using Lemma 5.2, and the well known weak* continuity properties of determinants in the Sobolev space $W^{1, \infty}$ (see for instance [12]), we have

$$
\begin{aligned}
\partial N\left(f_{0}, f_{1}\right) & =N\left(1, f_{0}, f_{1}\right) \\
& =\sum_{i=0}^{+\infty} \int_{K_{i}} \theta_{i}(s, t) \operatorname{det}\left(\begin{array}{cc}
\partial_{s} f_{0}\left(\varphi_{i}(s, t)\right), & \partial_{t} f_{0}\left(\varphi_{i}(s, t)\right) \\
\partial_{s} f_{1}\left(\varphi_{i}(s, t)\right), & \partial_{t} f_{1}\left(\varphi_{i}(s, t)\right)
\end{array}\right) d s d t= \\
& =\sum_{i=0}^{+\infty} \lim _{\varepsilon \rightarrow 0^{+}} \int_{K_{i}} \theta_{i}(s, t) \operatorname{det}\left(\begin{array}{cc}
\partial_{s} f_{0}\left(\varphi_{i}^{\varepsilon}(s, t)\right), & \partial_{t} f_{0}\left(\varphi_{i}^{\varepsilon}(s, t)\right) \\
\partial_{s} f_{1}\left(\varphi_{i}^{\varepsilon}(s, t)\right), & \partial_{t} f_{1}\left(\varphi_{i}^{\varepsilon}(s, t)\right)
\end{array}\right) d s d t .
\end{aligned}
$$


Since we are dealing with normal currents it results $\theta_{i} \in B V\left(K_{i}\right)$ while, by convolution approximation, we may assume that the compositions $\left.f_{k}\left(\varphi_{i}^{\imath}(s, t)\right)\right)$ are $\mathcal{C}^{2}$ functions. Integrating by parts the $i$-terms of the above expression we obtain

$$
\int_{K_{i}} \theta_{i}(s, t)\left[\partial_{s} f_{0}\left(\varphi_{i}^{\varepsilon}(s, t)\right) \partial_{t} f_{1}\left(\varphi_{i}^{\varepsilon}(s, t)\right)-\partial_{t} f_{0}\left(\varphi_{i}^{\varepsilon}(s, t)\right) \partial_{s} f_{1}\left(\varphi_{i}^{\varepsilon}(s, t)\right)\right] d s d t=
$$

$=\int_{c_{i}}^{d_{i}}\left[\theta_{i} f_{0} \partial_{t} f_{1}\right]_{s=a_{i}}^{s=b_{i}} d t-\int_{a_{i}}^{b_{i}}\left[\theta_{i} f_{0} \partial_{s} f_{1}\right]_{t=c_{i}}^{s=d_{i}} d s-\int_{K_{i}} f_{0} d\left[\partial_{s}\left(\theta_{i} \partial_{t} f_{1}\right)-\partial_{t}\left(\theta_{i} \partial_{s} f_{1}\right)\right](s, t)=$

$=\int_{c_{i}}^{d_{i}}\left[\theta_{i} f_{0} \partial_{t} f_{1}\right]_{s=a_{i}}^{s=b_{i}} d t-\int_{a_{i}}^{b_{i}}\left[\theta_{i} f_{0} \partial_{s} f_{1}\right]_{t=c_{i}}^{s=d_{i}} d s+\int_{K_{i}} f_{0} \partial_{s} f_{1} \partial_{t} \theta_{i}(s, t)-\int_{K_{i}} f_{0} \partial_{t} f_{1} \partial_{s} \theta_{i}(s, t)$.

Passing to the operator for the 1-currents in (5.5) we have

(5.6) $\widetilde{\partial N}(\omega)=\sum_{i=0}^{+\infty}\left[\int_{c_{i}}^{d_{i}}\left[\theta_{i} \int_{M}\left\langle\omega, v_{2, i}\right\rangle d \varphi_{i}\right]_{s=a_{i}}^{s=b_{i}} d t-\int_{a_{i}}^{b_{i}}\left[\theta_{i} \int_{M}\left\langle\omega, v_{1, i}\right\rangle d \varphi_{i}\right]_{t=c_{i}}^{t=d_{i}} d s+\right.$

$$
\left.+\int_{K_{i}}\left(\int_{M}\left\langle\omega, v_{1, i}\right\rangle d \varphi_{i}\right) \partial_{t} \theta_{i}(s, t)-\int_{K_{i}}\left(\int_{M}\left\langle\omega, v_{2, i}\right\rangle d \varphi_{i}\right) \partial_{s} \theta_{i}(s, t)\right] .
$$

On the other hand, integrating by parts in (5.3) we get

$$
\begin{aligned}
\tilde{N}(d \omega) & =-\sum_{i=0}^{+\infty} \lim _{\varepsilon \rightarrow 0^{+}} \int_{K_{i}} \theta_{i}(s, t)\left[\frac{\partial}{\partial t} \int_{M}\left\langle\omega, v_{1, i}\right\rangle d \varphi_{i}^{\varepsilon}(s, t)-\frac{\partial}{\partial s} \int_{M}\left\langle\omega, v_{2, i}\right\rangle d \varphi_{i}^{\varepsilon}(s, t)\right] d s d t= \\
& =\sum_{i=0}^{+\infty} \lim _{\varepsilon \rightarrow 0^{+}}\left[\int_{c_{i}}^{d_{i}}\left[\theta_{i} \int_{M}\left\langle\omega, v_{2, i}\right\rangle d \varphi_{i}^{\varepsilon}\right]_{s=a_{i}}^{s=b_{i}} d t-\int_{a_{i}}^{b_{i}}\left[\theta_{i} \int_{M}\left\langle\omega, v_{1, i}\right\rangle d \varphi_{i}^{\varepsilon}\right]_{t=c_{i}}^{t=d_{i}} d s+\right. \\
& \left.+\int_{K_{i}}\left(\int_{M}\left\langle\omega, v_{1, i}\right\rangle d \varphi_{i}^{\varepsilon}\right) \partial_{t} \theta_{i}(s, t)-\int_{K_{i}}\left(\int_{M}\left\langle\omega, v_{2, i}\right\rangle d \varphi_{i}^{\varepsilon}\right) \partial_{s} \theta_{i}(s, t)\right] .
\end{aligned}
$$


By standard properties of convolution kernels and since $\varphi_{i}^{\varepsilon}$ are equi-Lipschitz, passing to the limit as $\varepsilon \rightarrow 0^{+}$under the integral sign, it follows

$$
\begin{array}{r}
\widetilde{N}(d \omega)=\sum_{i=0}^{+\infty}\left[\int_{c_{i}}^{d_{i}}\left[\theta_{i} \int_{M}\left\langle\omega, v_{2, i}\right\rangle d \varphi_{i}\right]_{s=a_{i}}^{s=b_{i}} d t-\int_{a_{i}}^{b_{i}}\left[\theta_{i} \int_{M}\left\langle\omega, v_{1, i}\right\rangle d \varphi_{i}\right]_{t=c_{i}}^{t=d_{i}} d s+\right. \\
\left.\quad+\int_{K_{i}}\left(\int_{M}\left\langle\omega, v_{1, i}\right\rangle d \varphi_{i}\right) \partial_{t} \theta_{i}(s, t)-\int_{K_{i}}\left(\int_{M}\left\langle\omega, v_{2, i}\right\rangle d \varphi_{i}\right) \partial_{s} \theta_{i}(s, t)\right] .
\end{array}
$$

Hence, by inspection of (5.6) and (5.7) we get $\widetilde{N}(d \omega)=\widetilde{\partial N}(\omega)$. In such a way, if $T-S=\partial N$ in $\mathcal{P}_{p}(M)$, passing to the $\sim$ operator we obtain

$$
\widetilde{T}(\omega)-\widetilde{S}(\omega)=\widetilde{\partial N}(\omega)=\widetilde{N}(d \omega)
$$

for every 1-form $\omega$. Therefore, to conclude that two homologous metric 1-currents on $\mathcal{P}_{p}(M)$ are in fact homologous as euclidean currents one need just to prove that the right hand side of (5.3) vanishes along closed 1 -forms.

\section{2 - Metric homology of $\mathcal{P}_{p}\left(T^{n}\right)$.}

On the flat torus " $T^{n}$ the above question has some answers, while for a general manifold it seems that a better knowledge of the geometry of $\mathcal{P}_{p}(M)$ would be useful. Indeed, it is known that the homology $H_{1}\left({ }^{(} T^{n}, \mathbb{R}\right)$ and the cohomology $H^{1}\left(T^{n}, \mathbb{R}\right)$ are $n$-dimensional vector spaces. Moreover, the cohomology on ${ }^{\prime n}$ is generated by the classes $\left[d x_{1}\right],\left[d x_{2}\right], \ldots,\left[d x_{n}\right]$. In other words, for every closed 1-forms $\omega$ on ' $T^{n}$ there exists $P \in \mathbb{R}^{n}$ and $f \in \mathcal{C}^{1}\left(T^{n}\right)$ such that $\omega=P+d f$. For details we refer for instance to [14, 23]. Therefore, the right hand side of (5.3) vanishes along closed 1-forms $\omega$ if, as a vector of $\mathbb{R}^{n}$, it results

$$
\sum_{i=0}^{+\infty} \lim _{\varepsilon \rightarrow 0^{+}} \int_{K_{i}} \theta_{i}(s, t)\left(\frac{\partial}{\partial t} \int_{T^{m}} v_{1} d \varphi_{i}^{\varepsilon}(s, t)-\frac{\partial}{\partial s} \int_{T^{n}} v_{2} d \varphi_{i}^{\varepsilon}(s, t)\right) d s d t=0 .
$$

Indeed, since every closed form is represented by $P+d f$, it suffices to check that the right hand side of (5.3) evaluated on $d f$ is equal to zero. For 
if, by Lemma 4.2 it results

$$
\begin{aligned}
\int_{K_{i}} \theta_{i}(s, t)\left(\frac{\partial}{\partial t} \int_{\mathbb{T}^{n}}\left\langle d f, v_{1}\right\rangle d \varphi_{i}^{\varepsilon}(s, t)-\frac{\partial}{\partial s} \int_{\mathbb{T}^{n}}\left\langle d f, v_{2}\right\rangle d \varphi_{i}^{\varepsilon}(s, t)\right) d s d t= \\
=\int_{K_{i}} \theta_{i}(s, t)\left(\frac{\partial^{2}}{\partial t \partial s} \int_{\mathbb{T}^{n}} f d \varphi_{i}^{\varepsilon}-\frac{\partial^{2}}{\partial s \partial t} \int_{T^{n}} f d \varphi_{i}^{\varepsilon}\right) d s d t
\end{aligned}
$$

Denoting by $g(s, t)=\int_{T^{n}} f d \varphi_{i}^{\varepsilon}$, approximating $g$ by $g_{n}=\rho_{n} * g$, where $\rho_{n}$ are standard convolution kernels, the above integral is equal to

$$
\begin{aligned}
\int_{K_{i}} \theta_{i}(s, t)\left(\frac{\partial^{2}}{\partial t \partial s} g(s, t)-\frac{\partial^{2}}{\partial s \partial t} g(s, t)\right) d s d t= \\
\quad=\lim _{n \rightarrow+\infty} \int_{K_{i}} \theta_{i}(s, t)\left(\frac{\partial^{2}}{\partial t \partial s} g_{n}(s, t)-\frac{\partial^{2}}{\partial s \partial t} g_{n}(s, t)\right) d s d t=0 .
\end{aligned}
$$

Hence we get the following

Definition 5.3. We say that $N \in \boldsymbol{I}_{2}^{\text {reg }}\left(\mathcal{P}_{p}\left(T^{n}\right)\right)$ if $N$ is a regular metric 2current and the bi-Lipschitz curves $\varphi_{i}$ of its parametric representation satisfy the compatibility condition (5.8). Moreover we say that $S, T \in \boldsymbol{I}_{1}^{c}\left(T^{n}\right)$ are homologous on $\mathcal{P}_{p}\left(T^{n}\right)$ if there exists $N \in \boldsymbol{I}_{2}^{\text {reg }}\left(\mathcal{P}_{p}\left(T^{n}\right)\right)$ such that $S-T=\partial N$.

The above definition makes sense since on the flat torus we can recover the homology classes by homotopy. Actually we have the following

Lemma 5.4. Let $\gamma, \eta:[0,1] \rightarrow T^{n}$ be two smooth closed curves on $T^{n}$. Then the metric closed 1-currents $T_{\rho}$ and $T_{\sigma}$, where $\rho_{t}=\delta_{\gamma(t)}, \sigma_{t}=\delta_{\eta(t)}$, are homologous on $\mathcal{P}_{p}\left(T^{n}\right)$ if and only if $\gamma$ and $\eta$ are smooth homologous curves on ${ }^{n} T^{n}$.

Proof. Fix $x_{0}$ a base point for the fundamental homotopy group $\pi_{1}\left(T^{n}, x_{0}\right) \approx H_{1}\left(T^{n}\right)$. Therefore, if $\gamma, \eta:[0,1] \rightarrow T^{n}$ are two smooth homologous closed curves there exists a smooth homotopy $H:[0,1] \times$ 
$\times[0,1] \rightarrow T^{n}$ such that

$$
H(s, 1)=H(s, 0)=x_{0}, \quad H(1, t)=\gamma(t), \quad H(0, t)=\eta(t) .
$$

Consider the Lipschitz function $\varphi(s, t)=\delta_{H(s, t)}$. It turns out that $v_{1}=\partial_{s} H(s, t), v_{2}=\partial_{t} H(s, t)$. Therefore, the curve $\varphi$ is regular. Moreover, the compatibility condition (5.8) amounts to $\partial_{s, t}^{2} H=\partial_{s, t}^{2} H$. Therefore the metric 2-current $N$ associated to the Lipschitz function $\varphi$ belongs to $\boldsymbol{I}_{2}^{\text {reg }}\left(\mathcal{P}_{p}\left(T^{n}\right)\right)$. We compute

$$
\begin{aligned}
\partial N\left(f_{0}, f_{1}\right) & =N\left(1, f_{0}, f_{1}\right)= \\
& =\int_{0}^{1} \int_{0}^{1}\left(\partial_{s} f_{0}(\varphi(s, t)) \partial_{t} f_{1}(\varphi(s, t))-\partial_{t} f_{0}(\varphi(s, t)) \partial_{s} f_{1}(\varphi(s, t))\right) d s d t= \\
& =\int_{0}^{1}\left[f_{0}\left(\varphi(s, t) \partial_{t} f_{1}(\varphi(s, t))\right]_{s=0}^{s=1} d t-\int_{0}^{1}\left[f_{0}\left(\varphi(s, t) \partial_{s} f_{1}(\varphi(s, t))\right]_{t=0}^{t=1} d s=\right.\right. \\
& =\int_{0}^{1}\left[f_{0}(\varphi(1, t)) \partial_{t} f_{1}(\varphi(1, t))-f_{0}(\varphi(0, t)) \partial_{t} f_{1}(\varphi(0, t))\right] d t= \\
& =\int_{0}^{1}\left[f_{0}\left(\rho_{t}\right) \partial_{t} f_{1}\left(\rho_{t}\right)-f_{0}\left(\sigma_{t}\right) \partial_{t} f_{1}\left(\sigma_{t}\right)\right] d t=T_{\rho}\left(f_{0}, f_{1}\right)-T_{\sigma}\left(f_{0}, f_{1}\right) .
\end{aligned}
$$

Hence, $T_{\sigma}$ and $T_{\rho}$ are homologous on $\mathcal{P}_{p}\left(T^{n}\right)$. On the other hand, since for $\rho_{t}=\delta_{\gamma(t)}$ one has

$$
\widetilde{T_{\rho}}(\omega)=\int_{0}^{1}\langle\omega(\gamma(t)), \dot{\gamma}(t)\rangle d t,
$$

if $T_{\rho}$ and $T_{\sigma}$ are homologous on $\mathcal{P}_{p}\left(T^{n}\right)$ then, because of the compatibility condition, we have

$$
T_{\rho}-T_{\sigma}=\partial N \Rightarrow \widetilde{T_{\rho}}(\omega)-\widetilde{T_{\sigma}}(\omega)=\widetilde{\partial N}(\omega)=\widetilde{N}(d \omega)=0 .
$$

Therefore $\gamma$ and $\eta$ are homologous on ${ }^{\prime n}{ }^{n}$ as well.

Lemma 5.4 ensures that $\mathcal{P}_{p}\left(T^{n}\right)$ has at least $n$ distinct homology classes. Therefore, it make sense to address the question to recover mass minimizing currents on $\mathcal{P}_{p}\left(\mathbb{T}^{n}\right)$ by knowing the structure of the Eulerian 
representations of Mather's measures on the underlying space ' $T{ }^{n}$. A remarkable point in this question is that by changing the exponent $p$ the Eulerian representations on the manifold $M$ are unchanged, i.e. the minimization problem $\mathbf{P}$ does not depend on the exponent $p$ of the Lagrangian $L(x, v)=|v|^{p}$. However, in general we don't know if an analogous result could be true at the level of the Wasserstein space $\mathcal{P}_{p}(M)$ since, by changing the exponent $p$, we are changing the metric of the Wasserstein space, and then it's geodesic structure is in general modified. In the next Section we show how, for this special choice of the Lagrangian, some information on the underlying space $M$ can be transpose at the level of the Wasserstein space $\mathcal{P}_{p}(M)$.

5.3 - Mass minimizing metric currents on $\mathcal{P}_{p}\left({ }^{(T}{ }^{n}\right)$ and Eulerian representations.

Let $(\rho, v)$ be an Eulerian representation of a Mather's minimal measure on the flat torus ${ }^{2}{ }^{n}$. We claim that the current $T_{\rho}$ is a mass minimizing metric 1-current of $\mathcal{P}_{p}\left({ }^{(} T^{n}\right)$ in its homology class. Indeed, it results that $T_{\rho} \in \mathcal{I}_{1}^{c}\left(\mathcal{P}_{p}\left(T^{n}\right)\right)$. Since $(\rho, v)$ solves problem $\mathbf{P}$ and such problem with the Lagrangian $L(x, v)=|v|^{p}$ is equivalent to minimize the mass (to the power $p$ ) of currents in the corresponding homology class (see Proposition 3.7 of [11]), for every 1-currents $T$ belonging to $\left[T_{\rho, v}\right]$ it results

$$
\int_{0}^{1} \int_{T^{n}}|v(x, t)|^{p} d \rho_{t} d t=\mathcal{K}(\rho, v) \leq M(T)^{p} .
$$

Therefore, if $S=\sum_{i=1}^{+\infty} T_{\theta_{i}, \rho_{i}} \in \mathcal{I}_{1}^{c}\left(\mathcal{P}_{p}\left(T^{n}\right)\right)$ is homologous to $T_{\rho}$, by using Theorem 2.8 and Lemma 4.1 we compute

$$
\begin{aligned}
& M\left(T_{\rho}\right)^{p}=\left(\int_{0}^{1}|\dot{\rho}|_{p} d t\right)^{p} \leq \int_{0}^{1}|\dot{\rho}|_{p}^{p} d t=\int_{0}^{1} \int_{T^{n}}|v(x, t)|^{p} d \rho_{t} d t \leq M(\tilde{S})^{p} \leq \\
& \leq\left(\sum_{i=1}^{+\infty} \int_{a_{i}}^{b_{i}}\left|\theta_{i}\right|\left(\int_{T^{n}}\left|v_{i}\right| d \rho_{i}\right) d t\right)^{p} \leq\left(\sum_{i=1}^{+\infty} \int_{a_{i}}^{b_{i}}\left|\theta_{i}\right|\left(\int_{T^{n}}\left|v_{i}\right|^{p} d \rho_{i}\right)^{1 / p} d t\right)^{p}= \\
& =\left(\sum_{i=1}^{+\infty} \int_{a_{i}}^{b_{i}}\left|\theta_{i}\right|\left|\dot{\rho}_{i}\right|_{p} d t\right)^{p}=\left(\sum_{i=1}^{+\infty} M\left(T_{\theta_{i}, \rho_{i}}\right)\right)^{p}=M(S)^{p},
\end{aligned}
$$


where we used also Jensen and Hölder inequality. Actually, the above computations hold for any manifold $M$ as well. The matter is that for a general manifold $M$ we are not able to recover distinct metric homology classes of $\mathcal{P}_{p}(M)$. Moreover, it will be very interesting to study the above minimization property for more general Lagrangian $L(x, v)$. Actually, the choice of $L(x, v)=|v|^{p}$ is crucial to relate the mass of a metric current to the action of the Eulerian representation by using the continuity equation. Moreover, the continuity equation is the key tool to consider the $\sim$ operator. The matter is that for general Lagrangian the use of the continuity equation seems to be compromised. Therefore, we believe that any effort in this direction necessarily would involve new ideas and different techniques than those used in this paper.

\section{4 - Some minimal Eulerian representation on $T^{2}$.}

In this subsection we work out in detail an example of explicit minimal measure on the flat 2 -torus.

This example will be better described with the help of the Figure 5.1. By $\left\{\gamma_{i}\right\}_{i=1, \ldots, 4}$ we denote both the curves and their supports. The distinction will be clear from the context.

Let $\left\{\gamma_{i}\right\}_{i=1, \ldots, 4}$ be as in Figure 5.1.

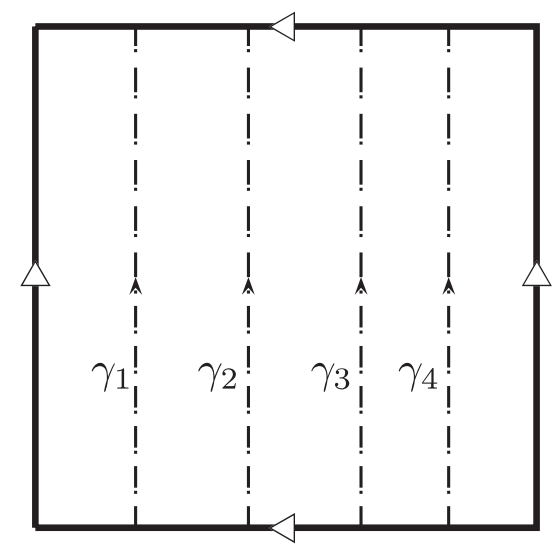

Fig. 5.1. An example of path on the torus.

Consider $\mu \in \mathcal{P}\left(T^{r} \mathbb{T}^{2}\right)$ defined by $\mu=\frac{1}{4}\left(\left(\mathcal{H}^{1}\left\llcorner\gamma_{1}\right) \otimes \delta_{e_{2}}+\left(\mathcal{H}^{1}\left\llcorner\gamma_{2}\right) \otimes \delta_{e_{2}}+\left(\mathcal{H}^{1}\left\llcorner\gamma_{3}\right) \otimes \delta_{e_{2}}+\left(\mathcal{H}^{1}\left\llcorner\gamma_{4}\right) \otimes \delta_{e_{2}}\right)\right.\right.\right.\right.$. 
The measure $\mu$ is invariant for the geodesic flow, the homology class of $\mu$ is $[\mu]=(0,1) \in \mathbb{R} \times \mathbb{R}=H_{1}\left(T^{2}\right)$, and $\mu$ is minimal being its cost equal to the length of the minimal geodesic in this class. It turns out that every curve of measures of the form

$$
\rho(t)=\frac{1}{4}\left(\mu _ { 1 } \left\llcorner\gamma_{1}+\mu_{2}\left\llcorner\gamma_{2}+\mu_{3}\left\llcorner\gamma_{3}+\mu_{4}\left\llcorner\gamma_{4}\right), \quad v(x, t)=e_{2}\right.\right.\right.\right.
$$

is a minimal Eulerian representation of $\mu$, where $\mu_{i}(i=1, \ldots, 4)$ is any probability measures on $\mathbb{S}^{1}$ concentrated on $\gamma_{i}$.

Acknowledgments. The author wishes to thank L. De Pascale and M. S. Gelli for useful discussions on the subject. The author is also grateful to L. Ambrosio for careful reading and precious comments on previous versions of this paper.

\section{REFERENCES}

[1] L. Ambrosio, Lecture Notes on Transport Problems, in "Mathematical Aspects of Evolving Interfaces". Lecture Notes in Mathematics, 1812 (Springer, Berlin, 2003), pp. 1-52.

[2] L. Ambrosio - N. Gigli - G. Savaré, Gradient Flows in Metric Spaces and in the Space of Probability Measures, Lectures in Mathematics ETH Zurich, Birkhauser Verlag, Basel, 2005.

[3] L. Ambrosio - B. Kirchneim, Currents in Metric Spaces, Acta Mathematica, 185 , no. 1 (2000), pp. $1-80$.

[4] L. Ambrosio - P. Tilli, Topics on Analysis in Metric Spaces, Oxford Lectures Series in Mathematics and its Applications, 25, Oxford University Press, Oxford, 2004.

[5] V. BANGert, Minimal measures and minimizing closed normal one-currents, GAFA Geom. Funct. Anal., 9, no. 3 (1999), pp. 413-427.

[6] G. BouchittÉ - G. Buttazzo, Characterization of optimal shapes and masses through Monge-Kantorovich equation, Journal European Math. Soc., 3 (2001), pp. 139-168.

[7] Y. BREnIER, Extended Monge-Kantorovich Theory, in Optimal Transportation and Applications, Lecture Notes in Mathematics, 1813 (Springer, Berlin, 2003), pp. 91-121.

[8] J. Benamou - Y. Brenier, A computational fluid mechanics solution to the Monge-Kantorovich mass transfer problem, Numer. Math., 84, no. 3 (2000), pp. 375-393.

[9] P. Bernard - B. Buffoni, Optimal mass transportation and Mather theory, J. Eur. Math. Soc. (JEMS), 9, no. 1 (2007), pp. 85-121.

[10] G. Contreras - R. Iturriaga, Global Minimizers of Autonomous Lagrangians. IMPA, Rio de Janeiro, 1999.

[11] L. De Pascale - M. S. Gelli - L. Granieri, Minimal measures, onedimensional currents and the Monge-Kantorovich problem, Calc. Var., 27, no. 1 (2006), pp. 1-23. 
[12] B. Dacorogna, Direct Methods in the Calculus of Variations, second edition, Springer, 2008.

[13] L. C. Evans, Partial differential equations and Monge-Kantorovich mass transfer (survey paper), Current Developments in Mathematics, 1997, International Press (1999), edited by S. T. Yau.

[14] W. Fulton, Algebraic Topology. Springer, 1995.

[15] H. Federer, Geometric Measure Theory. Springer (Berlin), 1969.

[16] W. GangBo - H. KIL - T. PACInI, Differential forms on Wasserstein space and infinite-dimensional Hamiltonian systems, forthcoming on Memoirs AMS.

[17] W. GANGBO - R. J. MC CANN, The geometry of optimal transportation, Acta Math., 177 (1996), pp. 113-161.

[18] M. Giaquinta - G. ModicA - J. SoučEK, Cartesian currents in the calculus of variations. I. Cartesian currents. Ergebnisse der Mathematik und ihrer Grenzgebiete. 3. Folge. A Series of Modern Surveys in Mathematics 37. Springer-Verlag, Berlin, 1998.

[19] L. GRANIERI, On action minimizing measures for the Monge-Kantorovich problem, NoDEA 14 (2007), pp. 125-152.

[20] L. Granieri, Mass Transportation Problems and Minimal Measures. Ph.D. Thesis in Mathematics, Pisa, 2005.

[21] B. Kloeckner, Geometric study of Wasserstein spaces: Euclidean spaces, Annali della Scuola Normale Superiore di Pisa, Classe di Scienze IX, 2 (2010), pp. 297-323.

[22] R. JORDAN - D. KINDERLEHRER - F. OTTO, The variational formulation of the Fokker-Plank equation, Siam J. Math. Anal., 29 (1998), pp. 1-17.

[23] J. Jost, Riemannian Geometry and Geometric Analysis. Springer, 2002.

[24] J. Jost, Nonpositive Curvature: Geometric and Analytic Aspects. Lectures in Math. ETH Zurich, Birkhauser Verlag, Basel, 1997.

[25] U. LANG - V. SCHROEDER, Kirszbraun's theorem and metric spaces of bounded curvature, GAFA Geom. Funct. Anal., 7 (1997), pp. 535-560.

[26] F. Отто, The geometry of dissipative evolution equations: the porus medium equation, Comm. Partial Differential Equations 26, no. 1-2 (2001), pp. 101-174.

[27] K. T. Sturm, Stochastics and Analysis on Metric Spaces, lecture notes in preparation.

[28] K. T. STURM, Metric spaces of lower bounded curvature, Exposition. Math., 17, no. 1 (1999), pp. 35-47.

[29] K. T. STuRM, Probability Measures on Metric Spaces of Nonpositive Curvature, Heat Kernels and Analysis on Manifolds, Graphs, and Metric Spaces (Paris, 2002), pp. 357-390, Contemp. Math., 338, AMS, Providence, RI, 2003.

[30] K. T. STURM, On the geometry of metric measure spaces. I, Acta Math., 196, no.1 (2006), pp. 65-131.

[31] C. Villani, Topics in Mass Transportation. Graduate Studies in mathematics, 58, AMS, Providence, RI, 2003.

[32] C. Villani, Optimal Transport, Old and New. Springer, 2009.

[33] S. WENGER, Isoperimetric inequalities of euclidean type in metric spaces, GAFA, Geom. funct. anal., Vol. 15 (2005), pp. 534-554.

[34] S. WEnGER, Flat convergence for integral currents in metric spaces, Calc. Var., 28 (2007), pp. 139-160.

Manoscritto pervenuto in redazione il 28 settembre 2009. 
